\title{
Joint D2D Group Association and Channel Assignment in Uplink Multi-Cell NOMA Networks: A Matching-Theoretic Approach \\ DOI:
}

10.1109/TCOMM.2019.2944142

\section{Document Version}

Accepted author manuscript

Link to publication record in Manchester Research Explorer

Citation for published version (APA):

Baidas, M. W., Bahbahani, M. S., Alsusa, E., Hamdi, K., \& Ding, Z. (2019). Joint D2D Group Association and Channel Assignment in Uplink Multi-Cell NOMA Networks: A Matching-Theoretic Approach. I E E Transactions on Communications. https://doi.org/10.1109/TCOMM.2019.2944142

\section{Published in:}

I E E E Transactions on Communications

\section{Citing this paper}

Please note that where the full-text provided on Manchester Research Explorer is the Author Accepted Manuscript or Proof version this may differ from the final Published version. If citing, it is advised that you check and use the publisher's definitive version.

\section{General rights}

Copyright and moral rights for the publications made accessible in the Research Explorer are retained by the authors and/or other copyright owners and it is a condition of accessing publications that users recognise and abide by the legal requirements associated with these rights.

\section{Takedown policy}

If you believe that this document breaches copyright please refer to the University of Manchester's Takedown Procedures [http://man.ac.uk/04Y6Bo] or contact uml.scholarlycommunications@manchester.ac.uk providing relevant details, so we can investigate your claim.

\section{OPEN ACCESS}




\title{
Joint D2D Group Association and Channel Assignment in Uplink Multi-Cell NOMA Networks: A Matching-Theoretic Approach
}

\author{
Mohammed W. Baidas $\dagger$, Mohammed S. Bahbahani†, Emad Alsusa $\S$, Khairi A. Hamdi $\S$, and Zhiguo Ding $\S$ \\ $\dagger$ Department of Electrical Engineering, College of Engineering and Petroleum, Kuwait University, Kuwait \\ $\ddagger$ Department of Electronics Engineering Technology, Public Authority of Applied Education and Training, Kuwait \\ $\S$ School of Electrical and Electronic Engineering, University of Manchester, Manchester, United Kingdom \\ (Email: m.baidas@ku.edu.kw, ms.bahbhani@paaet.edu.kw, and \{e.alsusa, k.hamdi, zhiguo.ding\}@manchester.ac.uk)
}

\begin{abstract}
This paper studies joint device-to-device (D2D) group association and channel assignment in uplink multi-cell non-orthogonal multiple-access (NOMA) networks. Particularly, the goal is to assign D2D groups to cellular user channels at each base-station, while accounting for negative network externality due to the interference caused by pairing a user with a D2D group. To that end, a multi-objective signalto-interference-plus-noise ratio (SINR)-maximizing power allocation solution procedure is proposed to determine the optimal power allocation for each (D2D group, user) pair, while meeting quality-of-service (QoS) requirements. After that, the joint D2D group association and channel assignment problem is modeled as a student-project allocation with preferences over (student, project) pairs matching problem. More specifically, two polynomial-time complexity stable matching algorithms are proposed to pair D2D groups with users, and associate them with base-stations. Simulation results are presented to evaluate the proposed matching algorithms when combined with the devised solution procedure, and compare them to a joint D2D group association, channel assignment and power allocation (J-GA-CA-PA) scheme. More importantly, the proposed algorithms are shown to efficiently yield comparable SINR - per user and D2D receiver-to the J-GA-CA-PA scheme, while maintaining QoS requirements.
\end{abstract}

\section{Index Terms}

Channel assignment, device-to-device, matching, multi-cell, power allocation, quality-of-service

\section{INTRODUCTION}

Device-to-device (D2D) communications have recently received significant attention due to their ability to enhance spectral efficiency when operating in an underlay manner in cellular networks [1,2]. Specifically, D2D communication approaches have allowed cellular and D2Denabled devices to share spectrum resources, yielding improved energy and spectral efficiency

$\dagger$ Corresponding Author: Mohammed W. Baidas, Electrical Engineering Department, Kuwait University, PO Box: 5969, Safat, 13060, Kuwait City, Kuwait (Email: m.baidas@ku.edu.kw). 
gains over conventional cellular networks $[3,4]$. Hence, D2D communications are expected to significantly improve capacity and support massive connectivity of future 5G cellular networks [5]. On the other hand, an emerging multiple-access technique, namely non-orthogonal multipleaccess (NOMA), has recently been proposed to enhance spectral efficiency, reduce latency, and improve fairness and connectivity for next generation cellular networks by allowing multiple users to share the same spectrum simultaneously [6]. More specifically, NOMA allows several users to use the same resource block (e.g. time-slot, sub-carrier or spreading code) by exploiting channel gain differences through power-domain multiplexing. By utilizing successive interference cancellation (SIC), the throughput of users with poor channel conditions can concurrently be improved along with users with better channel conditions; ultimately improving the network throughput in comparison to orthogonal multiple access (OMA) [7]. Hence, incorporating NOMA into D2D communications allows D2D transmitters to share network resources to communicate with multiple D2D receivers in an underlaid fashion so as to enhance network sum-rate, while simultaneously serving cellular users. Most of the published literature on NOMA assumes singlecell networks; however, multi-cell scenarios are considered more realistic, and more importantly, more complex due to the interplay between multiple cells [8]. Thus, it is essential to consider resource allocation for underlaid D2D communications in multi-cell NOMA networks, while guaranteeing quality-of-service (QoS) for cellular users as well as D2D receivers.

Recently, several research works have considered resource allocation in single-cell NOMAbased D2D communications. For instance, [9] introduces the concept of a "D2D group", where a D2D transmitter (DT)—utilizing uplink NOMA transmission-communicates with multiple D2D receivers (DRs) simultaneously. Specifically, the target is to maximize network sum-rate by allowing multiple D2D groups to reuse the same user sub-channel, while satisfying signalto-interference-plus-noise (SINR) constraints for D2D as well as cellular users. The formulated problem happens to be NP-hard, and thus a many-to-one low-complexity matching algorithm is proposed to obtain a sub-optimal solution within a limited number of iterations. It has been demonstrated that the proposed NOMA-based D2D framework achieves near-optimal network sum-rate. In [10], NOMA-based D2D group communication is studied, where the DRs are ordered according to their QoS requirements, while incorporating two power allocation policies. It has been shown that the proposed QoS-based NOMA scheme yields lower outage probability than that with OMA. In [11], the authors study power control and channel assignment in singlecell D2D communication underlaying a NOMA cellular network, with the aim of maximizing 
the sum-rate of D2D pairs while guaranteeing QoS of the NOMA-based cellular users. Optimal power allocation conditions for cellular users are first derived, and then a dual-based iterative algorithm is proposed, yielding considerable D2D sum-rate gains over conventional OMA. User clustering and power assignment for a single-cell downlink NOMA network with underlay D2D users is considered in [12]. Specifically, the aim is to maximize network sum-rate by optimizing user clustering and power allocation, while providing interference protection to cellular users. The formulated problem is a mixed-integer non-convex problem, and thus is decomposed into two sub-problems. The first "user clustering" sub-problem is modeled as a matching game, and solved sequentially via two low-complexity algorithms. The second "power assignment" sub-problem is addressed using the complementary geometric programming and the arithmetic-geometric mean approximation, where a low-complexity solution is devised. After that, an iterative joint algorithm is proposed, and shown to achieve performance gains in terms of the average sum-rate in comparison to general NOMA, OFDMA, and D2D schemes. Joint sub-channel and power allocation for NOMA-enhanced D2D communication is considered in [13], where the objective is to maximize network sum-rate in an uplink single-cell NOMA network. The sub-channel assignment is formulated as a many-to-one matching problem, and a matching algorithm is devised. Additionally, sequential convex programming is applied to iteratively update power allocation to cellular users and D2D receivers. After that, joint sub-channel assignment and power allocation is performed, and shown to approach the exhaustive-search method in terms of network sum-rate.

It should be noted that matching theory has recently attracted much attention in the wireless literature for resource allocation in cellular networks. For instance, in [14], a novel approach for cell association is proposed based on the users' locations and proximity to the small-cell base-stations. Moreover, a stable user-cell association is obtained via a many-to-one deferred acceptance (DA) mechanism, which has been shown to reach a stable matching solution and achieve significant performance gains. In [15], the authors consider context-aware resource allocation in OFDMA-based small-cell networks, and formulate it as a two-sided one-to-one matching game between D2D-enabled user equipments (UEs) and resource blocks (RBs), while capturing both wireless and social metrics. The proposed self-organizing algorithm has been shown to offload larger amount of traffic than the context-unaware scheme, and with manageable complexity. In [16], joint spectrum allocation and power control for sum-rate maximization in NOMA-based heterogenous networks (HetNets) is considered. Specifically, the interaction 
between small base-stations (SBSs) and RBs is modeled as a two-sided many-to-one matching game. In addition, the power control at each SBS is solved iteratively via a sequential convex programming approach. It has been demonstrated that the proposed NOMA-based HetNets yield higher network sum-rate than conventional OMA-based HetNets. Joint sub-channel assignment, power allocation and user scheduling is studied in [17]. Particularly, a many-to-one two-sided user-subchannel stable matching game is proposed, which has been shown to outperform various orthogonal and non-orthogonal multiple access schemes.

This paper studies the problem of joint D2D group association and channel assignment in uplink multi-cell NOMA networks. Particularly, the aim is to associate D2D groups with basestations, and pair them with cellular users (with pre-assigned channels), such that the SINR of each user and D2D receiver satisfies QoS requirements. In turn, a joint D2D group association, channel assignment and power allocation (J-GA-CA-PA) scheme is formulated, and shown to be computationally-expensive. Therefore, an iterative solution procedure for multi-objective SINRmaximizing power allocation (SP-MO-SINR-MAX-PA) per (D2D group, user) pair is proposed, which takes into account the interference caused by each paired user at the D2D receivers, and also the interference caused by the D2D transmitter at each paired user. Moreover, the proposed solution procedure is utilized to determine the preference of each D2D group over the potential users within each cell, and also the preference of each base-station over the potential (D2D group, user) pairs. In turn, a stable matching solution based on the student-project allocation with preferences over (student, project) pairs $(S P A-(S, P))$ problem is considered [18]. Specifically, the lecturers are base-stations that have preferences over (D2D group, user) pairs, where the D2D groups resemble the students, while the users resemble the projects. Then, two stable matching algorithms with polynomial-time complexity are proposed, namely D2D group-oriented stable matching (DG-SM), and the base-station-oriented stable matching (BS-SM), which take into account the maximum number of D2D groups to be associated with each base-station [19]. Furthermore, the proposed DG-SM and BS-SM algorithms yield D2D group association and channel assignment stable matchings, such that no D2D group or base-stations would unilaterally change its association or channel assignment. Simulation results are presented to evaluate the performance of the proposed matching algorithms-when combined with the SP-MO-SINRMAX-PA - and compare them to the J-GA-CA-PA scheme. It is illustrated that the proposed algorithms are computationally-efficient, and yield comparable SINR — per cellular user and D2D receiver-to the J-GA-CA-PA scheme, while maintaining QoS requirements. 
To the best of the authors' knowledge, no prior work has applied the SPA problem with preferences of (D2D group, user) pairs, and augmented it with a multi-objective SINR-maximizing power allocation solution procedure for D2D-enabled uplink multi-cell NOMA networks. Hence, the main contributions of this work can be summarized as follows ${ }^{1}$ :

- Devised an iterative solution procedure to solve the multi-objective SINR-maximizing power allocation problem of each (D2D group, user) within each base-station.

- Modeled the D2D group association and channel assignment problem in uplink multi-cell NOMA networks as a SPA problem with preferences over (D2D group, user) pairs.

- Proposed two polynomial-time complexity stable matching algorithms to associate D2D groups with base-stations and pair them with user channels, so as to obtain D2D groupoptimal and base-station-optimal stable matching solutions.

- Formulated the J-GA-CA-PA problem, and compared it with the proposed stable matching algorithms, which are shown to efficiently (i.e. with low-complexity) associate D2D groups with base-stations and cellular user channels, and yield comparable SINR - per cellular user and D2D receiver-to the J-GA-CA-PA scheme, while maintaining QoS requirements.

In the rest of this paper, Section II presents the system model; while Section III outlines the joint D2D group association, channel assignment and power allocation problem formulation. The multi-objective SINR-maximizing power allocation per (D2D group, user) pair is devised in Section IV. The stable matching algorithms are proposed in Section V, while the simulation results are presented in Section VI. Finally, the conclusions are drawn in Section VII.

\section{SYSTEM MODEL}

In this section, the network model is presented along with the signal transmission model of the cellular users and D2D groups. Table I summarizes the main notations used in this paper.

\section{A. Network Model}

Consider an uplink multi-cell NOMA network with a set of $N$ cellular users $\mathcal{U}=\left\{U_{1}, \ldots, U_{n}, \ldots, U_{N}\right\}$, which are scattered within the coverage area of $Q$ base-stations (BSs). In particular, the set of BSs is denoted $\mathcal{B}=\left\{B S_{1}, \ldots, B S_{q}, \ldots, B S_{Q}\right\}$. Moreover, let $\mathcal{U}_{q} \subset \mathcal{U}$ be the subset of users associated with base-station $B S_{q}$. In addition, let $\mathcal{C}=\left\{C_{1}, \ldots, C_{n}, \ldots, C_{N}\right\}$ be the set of $N$

\footnotetext{
${ }^{1}$ A shorter version of this paper has been accepted for publishing in the Proceedings of the 2019 IEEE Wireless Communications and Networking Conference (WCNC) [20].
} 


\section{TABLE I}

NOTATIONS

\begin{tabular}{|c|c|}
\hline Symbol & Definition \\
\hline $\mathcal{B}$ & Set of base-stations \\
\hline $\mathcal{U}$ & Set of cellular users \\
\hline $\mathcal{D}$ & Set of D2D groups \\
\hline $\mathcal{C}$ & Set of orthogonal channels \\
\hline $\mathcal{U}_{q}$ & Subset of cellular users within coverage area of base-station $B S_{q}$ \\
\hline $\mathcal{D}_{q}$ & Subset of D2D groups within coverage area of base-station $B S_{q}$ \\
\hline $\mathcal{C}_{q}$ & Subset of channels per base-station $B S_{q}$ \\
\hline$Q$ & Number of base-stations in the network \\
\hline$N$ & Number of cellular users in the network \\
\hline$M$ & Number of D2D groups in the network \\
\hline$\xi_{q}$ & Maximum number of D2D groups per base-station $B S_{q}$ \\
\hline$D T_{m}$ & Transmitter of D2D group $D_{m}$ \\
\hline$D R_{m_{j}}$ & Receiver of D2D group $D_{m}$, for $j \in\{1,2\}$ \\
\hline$h_{n, q}$ & Channel coefficient of user $U_{n} \in \mathcal{U}_{q}$ and base-station $B S_{q}$ \\
\hline$h_{n, m_{t}, q}$ & Channel coefficient of $D T_{m}$ and $B S_{q}$ over channel $C_{n} \in \mathcal{C}_{q}$ \\
\hline$f_{n, m_{t}, m_{r_{j}}, q}$ & Channel coefficient of $D T_{m}$ and $D R_{m_{j}}$, for $j \in\{1,2\}$ over channel $C_{n} \in \mathcal{C}_{q}$ \\
\hline$z_{n, m_{r_{j}}, q}$ & Channel coefficient of user $U_{n} \in \mathcal{U}_{q}$ and $D R_{m_{j}}$, for $j \in\{1,2\}$ \\
\hline$a_{n, m_{j}, q}$ & Power allocation coefficient of the D2D receiver of group $D_{m}$, for $j \in\{1,2\}$ over channel $C_{n} \in \mathcal{C}_{q}$ \\
\hline$P$ & Total transmit power per channel \\
\hline$P_{n, q}$ & Transmit power of user $U_{n} \in \mathcal{U}_{q}$ \\
\hline$P_{n, m, q}$ & Transmit power of D2D group $D_{m} \in \mathcal{D}_{q}$ over channel $C_{n} \in \mathcal{C}_{q}$ \\
\hline $\mathcal{I}_{n, m, q}$ & Binary decision variable to indicate if $D_{m} \in \mathcal{D}_{q}$ is paired with $U_{n} \in \mathcal{U}_{q}$ \\
\hline$\gamma_{n, q}$ & SINR of user $U_{n} \in \mathcal{U}_{q}$ \\
\hline$\gamma_{n, m_{r_{j}}, q}$ & SINR at D2D receiver of group $D_{m} \in \mathcal{D}_{q}$, for $j \in\{1,2\}$ over channel $C_{n} \in \mathcal{C}_{q}$ \\
\hline $\bar{\gamma}_{T_{U}}$ & Target minimum SINR per user \\
\hline $\bar{\gamma}_{T_{D R}}$ & Target minimum SINR per D2D receiver \\
\hline
\end{tabular}

orthogonal channels that are assigned to the cellular users (i.e. $|\mathcal{U}|=|\mathcal{C}|$, where $|\cdot|$ denotes the cardinality of the parameter set). Also, let $\mathcal{C}_{q}$ be the set of channels available at $B S_{q}$, such that $\mathcal{C}_{1}, \ldots, \mathcal{C}_{q}, \ldots, \mathcal{C}_{Q}$ partition $\mathcal{C}$ (i.e. $\mathcal{C}_{q} \cap \mathcal{C}_{w}=\phi$ for $q \neq w$, and $\left.\bigcup_{q=1}^{Q} \mathcal{C}_{q}=\mathcal{C}\right)^{2}$. For convenience, let $C_{n} \in \mathcal{C}_{q}$ be the channel allocated to user $U_{n} \in \mathcal{U}_{q}$. Furthermore, it is assumed that there is a set of $M$ D2D groups, denoted $\mathcal{D}=\left\{D_{1}, \ldots, D_{m}, \ldots, D_{M}\right\}$, where each D2D transmitter $D T_{m}$ of D2D group $D_{m}$ communicates underlying the cellular network, by sending a superimposed

\footnotetext{
${ }^{2}$ User-base-station association and channel assignment is beyond the scope of this paper [21]. Also, no frequency reuse across multiple cells is assumed in this work, and hence, inter-cell interference is not considered. Alternatively, frequency channels could have been assigned to multiple users across the network cells, while ensuring sufficient physical separation, such that inter-cell interference becomes negligibly small [22]. Nevertheless, due to the close proximity of the D2D groups to the users, the resulting inter-user interference is much more dominant than inter-cell interference, and thus is considered in this work.
} 
signal to the receivers within its group (see Fig. 1a). For convenience, let $\mathcal{D}_{q} \subset \mathcal{D}$ be the set of D2D groups within the coverage area of $B S_{q}$. Since some D2D groups may fall within the overlapping region of multiple cells, then let $\xi_{q}$ be the maximum number of $\mathrm{D} 2 \mathrm{D}$ groups to be associated with a base-station $B S_{q}$, for $B S_{q} \in \mathcal{B}$. Additionally, the total transmit power per channel $C_{n} \in \mathcal{C}_{q}$ of each base-station $B S_{q}$ must not exceed $P, \forall B S_{q} \in \mathcal{B}$.

Remark 1: Without any loss of generality, and to reduce SIC complexity, delay and interference, it is assumed that there are two NOMA receivers within each D2D group $D_{m}$, namely $D R_{m_{1}}$ and $D R_{m_{2}}$, respectively, $\forall D_{m} \in \mathcal{D}$ (see Fig. 1b) [13]. More importantly, it is assumed that at most one D2D group $D_{m} \in \mathcal{D}_{q}$ can be assigned to any user channel $C_{n} \in \mathcal{C}_{q}, \forall B S_{q} \in \mathcal{B}$.
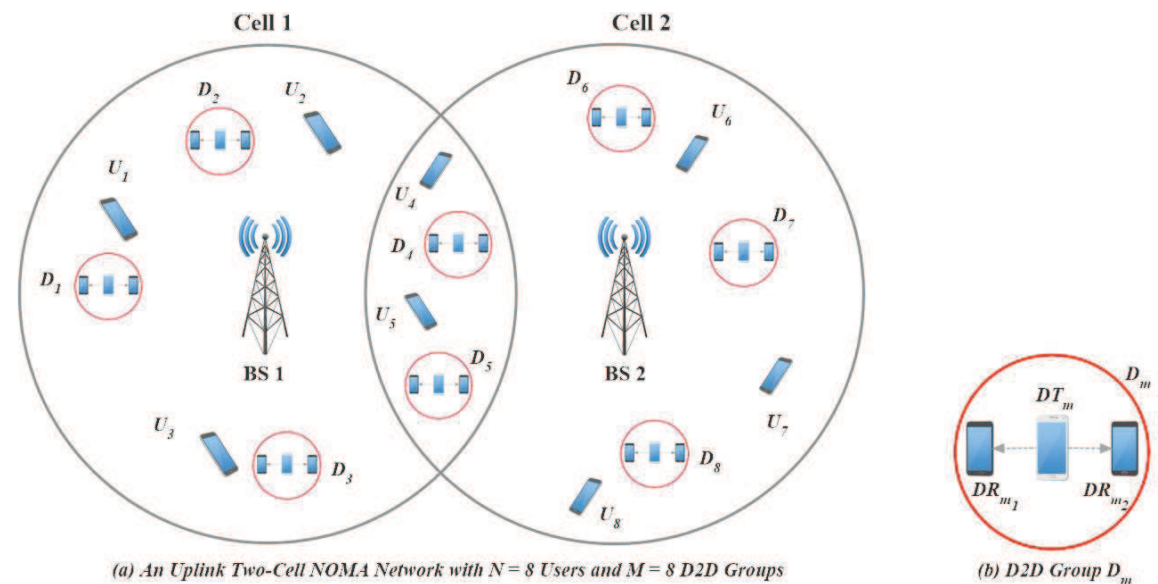

Fig. 1. (a) An Uplink Two-Cell NOMA Network with $N=8$ User and $M=8$ D2D Groups, and (b) D2D Group $D_{m}$

The instantaneous channel between cellular users, base-stations and D2D transmitters and receivers is assumed to follow narrowband Rayleigh fading with zero-mean $N_{0}$-variance additive white Gaussian noise (AWGN). Specifically, let $h_{n, q} \sim \mathcal{C N}\left(0, \sigma_{n, q}^{2}\right)$ and $h_{n, m_{t}, q} \sim$ $\mathcal{C N}\left(0, \sigma_{n, m_{t}, q}^{2}\right)$ be the channel coefficient of cellular user $U_{n} \in \mathcal{U}_{q}$ to $B S_{q}$ over channel $C_{n} \in \mathcal{C}_{q}$, and the channel coefficient of D2D transmitter $D T_{m}$ to $B S_{q}$ over channel $C_{n} \in \mathcal{C}_{q}$, respectively, with $\sigma_{n, q}^{2}=d_{n, q}^{-\nu}$ and $\sigma_{n, m_{t}, q}^{2}=d_{m_{t}, q}^{-\nu}$ being the corresponding channels gains; while $d_{n, q}$ and $d_{m_{t}, q}$ are the respective distances, and $\nu$ is the path-loss exponent. Similarly, let $f_{n, m_{t}, m_{r_{j}}, q} \sim \mathcal{C N}\left(0, \sigma_{n, m_{t}, m_{r_{j}}, q}^{2}\right)$ be the channel coefficient between D2D transmitter $D T_{m}$ and D2D receiver $D R_{m_{j}}$ (for $j \in\{1,2\}$ ) over channel $C_{n} \in \mathcal{C}_{q}$, with corresponding channel gain $\sigma_{n, m_{t}, m_{r_{j}}, q}^{2}=d_{m_{t}, m_{r_{j}}}^{-\nu}$, with $d_{m_{t}, m_{r_{j}}}$ being the respective distance. Lastly, let $z_{n, m_{r_{j}}, q} \sim$ $\mathcal{C N}\left(0, \sigma_{n, m_{r_{j}}, q}^{2}\right)$ be the channel coefficient between user $U_{n} \in \mathcal{U}_{q}$ and D2D receiver $D R_{m_{j}}$, such that $\sigma_{n, m_{r_{j}}, q}^{2}=d_{n, m_{r_{j}}}^{-\nu}$, for $j \in\{1,2\}$, with $d_{n, m_{r_{j}}}$ being the respective distance. 


\section{B. Transmission Model}

The received signal at $B S_{q}$ over channel $C_{n} \in \mathcal{C}_{q}$ is expressed as

$$
y_{n, q}=\sqrt{P_{n, q}} h_{n, q} x_{n, q}+\sum_{D_{m} \in \mathcal{D}_{q}} \mathcal{I}_{n, m, q} \sqrt{P_{n, m, q}} \chi_{n, m_{t}, q} h_{n, m_{t}, q}+\eta_{n, q},
$$

where $\eta_{n, q}$ is the AWGN sample at base-station $B S_{q}$, while $P_{n, q}$ and $P_{n, m, q}$ are the transmit powers of user $U_{n} \in \mathcal{U}_{q}$, and D2D group $D_{m} \in \mathcal{D}_{q}$, respectively. In addition, $x_{n, q}$ is the signal of user $U_{n} \in \mathcal{U}_{q}$, while $\chi_{n, m_{t}, q}$ is the transmitted superimposed signal by $D T_{m}$, written as

$$
\chi_{n, m_{t}, q}=\sqrt{a_{n, m_{r_{1}}, q}} x_{n, m_{r_{1}}, q}+\sqrt{a_{n, m_{2}, q}} x_{n, m_{r_{2}}, q}
$$

where $a_{n, m_{r_{1}}, q}+a_{n, m_{r_{2}}, q} \leq 1$. Moreover, $x_{n, m_{r_{1}}, q}$ and $x_{n, m_{r_{2}}, q}$ are the signals intended for D2D receivers $D R_{m_{1}}$ and $D R_{m_{2}}$, respectively, whereas $a_{n, m_{r_{1}}, q}$ and $a_{n, m_{r_{2}}, q}$ are the corresponding power allocation coefficients. On the other hand, $\mathcal{I}_{n, m, q}$ is a binary decision variable given by

$$
\mathcal{I}_{n, m, q}= \begin{cases}1, & \text { if D2D Group } D_{m} \text { is paired with user } U_{n} \in \mathcal{U}_{q}, \\ 0, & \text { otherwise. }\end{cases}
$$

In turn, the SINR of user $U_{n} \in \mathcal{U}_{q}$ is determined as [13]

$$
\gamma_{n, q}=\frac{P_{n, q}\left|h_{n, q}\right|^{2}}{\sum_{D_{m} \in \mathcal{D}_{q}} \mathcal{I}_{n, m, q} P_{n, m, q}\left|h_{n, m_{t}, q}\right|^{2}+N_{0}} .
$$

Remark 2: Since each user $U_{n} \in \mathcal{U}_{q}$ can be paired with at most one D2D group over channel $C_{n} \in \mathcal{C}_{q}$, then $\sum_{D_{m} \in \mathcal{D}_{q}} \mathcal{I}_{n, m, q} \leq 1$. In addition, the maximum number of D2D groups to be associated with a base-station $B S_{q}$ must satisfy $\sum_{U_{n} \in \mathcal{U}_{q}} \sum_{D_{m} \in \mathcal{D}_{q}} \mathcal{I}_{n, m, q} \leq \xi_{q}, \forall B S_{q} \in \mathcal{B}$. Lastly, a D2D group $D_{m} \in \mathcal{D}$ can only be paired to at most one user/channel across all base-stations (i.e. $\sum_{B S_{q} \in \mathcal{B}} \sum_{C_{n} \in \mathcal{C}_{q}} \mathcal{I}_{n, m, q} \leq 1$ ).

Remark 3: The total power constraint over each channel must be satisfied, according to $P_{n, q}+\sum_{D_{m} \in \mathcal{D}_{q}} \mathcal{I}_{n, m, q} P_{n, m, q} \leq P, \forall C_{n} \in \mathcal{C}_{q}$ and $\forall B S_{q} \in \mathcal{B}$.

The received signal at D2D receiver $D R_{m_{j}}$ (for $j \in\{1,2\}$ ) over $C_{n} \in \mathcal{C}_{q}$ is given by

$$
y_{n, m_{r_{j}}, q}=\sqrt{P_{n, m, q}} \chi_{n, m, q} f_{n, m_{t}, m_{r_{j}}, q}+\sqrt{P_{n, q}} x_{n, q} z_{n, m_{r_{j}}, q}+\eta_{n, m_{r_{j}}, q},
$$

where $\eta_{n, m_{r_{j}}, q}$ is the AWGN sample at $D R_{m_{j}}$. Based on the principle of NOMA, and for notational convenience, assume that $D R_{m_{2}}$ is the receiver with the worse channel conditions than $D R_{m_{1}}$. In turn, for $D R_{m_{2}}$ to be able to decode its signal, and for $D R_{m_{1}}$ to remove $D R_{m_{2}}$ 's signal and decode its own signal, then the following constraint must be satisfied $[11,13]$

$$
S_{n, m, q} \triangleq\left|f_{n, m_{t}, m_{r_{1}}, q}\right|^{2}\left(P_{n, q}\left|z_{n, m_{r_{2}}, q}\right|^{2}+N_{0}\right)-\left|f_{n, m_{t}, m_{r_{2}}, q}\right|^{2}\left(P_{n, q}\left|z_{n, m_{r_{1}}, q}\right|^{2}+N_{0}\right) \geq 0 .
$$


Thus, the SINR at $D R_{m_{2}}$ - when it decodes its own signal-is obtained as

$$
\gamma_{n, m_{r_{2}}, q}=\frac{\left|f_{n, m_{t}, m_{r_{2}}, q}\right|^{2} P_{n, m, q} a_{n, m_{r_{2}}, q}}{\left|f_{n, m_{t}, m_{r_{2}}, q}\right|^{2} P_{n, m, q} a_{n, m_{r_{1}}, q}+P_{n, q}\left|z_{n, m_{r_{2}}, q}\right|^{2}+N_{0}},
$$

while the resulting SINR of $D R_{m_{1}}$-when it decodes its own signal-is determined as

$$
\gamma_{n, m_{r_{1}}, q}=\frac{\left|f_{n, m_{t}, m_{r_{1}, q}}\right|^{2} P_{n, m, q} a_{n, m_{r_{1}}, q}}{P_{n, q}\left|z_{n, m_{r_{1}, q}, q}\right|^{2}+N_{0}} .
$$

Due to the interference caused by the $\mathrm{D} 2 \mathrm{D}$ transmitter $D T_{m}$ at the cellular user $U_{n}$, and by user $U_{n}$ at the D2D receivers $D R_{m_{j}}$ (for $j \in\{1,2\}$ ), it is essential to maintain quality-of-service (QoS) requirements at each cellular user and the associated D2D receivers, as

$$
\begin{aligned}
\gamma_{n, q} & \geq \bar{\gamma}_{T_{U}}, \quad \forall U_{n} \in \mathcal{U}_{q}, \forall B S_{q} \in \mathcal{B}, \\
\gamma_{n, m_{r_{j}}, q} & \geq \bar{\gamma}_{T_{D R}}, \quad \forall j \in\{1,2\}, \forall D_{m} \in \mathcal{D}_{q}, \forall U_{n} \in \mathcal{U}_{q}, \forall B S_{q} \in \mathcal{B},
\end{aligned}
$$

where $\bar{\gamma}_{T_{U}}$ and $\bar{\gamma}_{T_{D R}}$ are the target minimum SINR per user and D2D receiver, respectively.

\section{Joint D2D Group Association, Channel Assignment And Power Allocation \\ PROBLEM FORMULATION}

In this work, the aim is to jointly associate D2D groups with base-stations, assign them to user channels, and optimally allocate power. Moreover, the objective is to simultaneously maximize the SINR of each user $U_{n} \in \mathcal{U}_{q}$ and each D2D receiver $D R_{m_{j}}$ (for $j \in\{1,2\}$ ) subject to the target minimum SINR per user and D2D receiver. Thus, the joint D2D group association, channel assignment and power allocation (J-GA-CA-PA) problem is formulated as a multi-objective mixed-integer programming problem, as follows

\section{J-GA-CA-PA:}

$$
\begin{array}{ll}
\max & \left(\gamma_{U, B S},\left\{\gamma_{U, D R_{m_{1}}, B S}\right\}_{m=1}^{M},\left\{\gamma_{U, D R_{m_{2}}, B S}\right\}_{m=1}^{M}\right) \\
\text { s.t. } & P_{n, q}+\sum_{D_{m} \in \mathcal{D}_{q}} \mathcal{I}_{n, m, q} P_{n, m, q} \leq P, \quad \forall C_{n} \in \mathcal{C}_{q}, \forall B S_{q} \in \mathcal{B}, \\
& \sum_{D_{m} \in \mathcal{D}_{q}} \mathcal{I}_{n, m, q} \leq 1, \quad \forall C_{n} \in \mathcal{C}_{q}, \forall B S_{q} \in \mathcal{B}, \\
& \sum_{C_{n} \in \mathcal{C}_{q}} \sum_{D_{m} \in \mathcal{D}_{q}} \mathcal{I}_{n, m, q} \leq \xi_{q}, \quad \forall B S_{q} \in \mathcal{B}, \\
& \sum_{B S_{q} \in \mathcal{B}} \sum_{C_{n} \in \mathcal{C}_{q}} \mathcal{I}_{n, m, q} \leq 1, \quad \forall D_{m} \in \mathcal{D}, \\
& S_{n, m, q} \cdot \mathcal{I}_{n, m, q} \geq 0, \quad \forall D_{m} \in \mathcal{D}_{q}, \forall C_{n} \in \mathcal{C}_{q}, \forall B S_{q} \in \mathcal{B},
\end{array}
$$




$$
\begin{aligned}
& \left(\gamma_{n, q}-\bar{\gamma}_{T_{U}}\right) \cdot \sum_{D_{m} \in \mathcal{D}_{q}} \mathcal{I}_{n, m, q} \geq 0, \quad \forall U_{n} \in \mathcal{U}_{q}, \forall B S_{q} \in \mathcal{B} \\
& \left(\gamma_{n, m_{r_{j}}, q}-\bar{\gamma}_{T_{D R}}\right) \cdot \mathcal{I}_{n, m, q} \geq 0, \forall j \in\{1,2\}, \forall D_{m} \in \mathcal{D}_{q}, \forall C_{n} \in \mathcal{C}_{q}, \forall B S_{q} \in \mathcal{B} \\
& a_{n, m_{r_{1}}, q}+a_{n, m_{r_{2}}, q} \leq \mathcal{I}_{n, m, q}, \quad \forall D_{m} \in \mathcal{D}_{q}, \forall C_{n} \in \mathcal{C}_{q}, \forall B S_{q} \in \mathcal{B} \\
& 0 \leq P_{n, m, q} \leq P \cdot \mathcal{I}_{n, m, q}, \quad \forall D_{m} \in \mathcal{D}_{q}, \forall C_{n} \in \mathcal{C}_{q}, \forall B S_{q} \in \mathcal{B} \\
& P_{n, q} \geq 0, \quad \forall U_{n} \in \mathcal{U}_{q}, \forall B S_{q} \in \mathcal{B}, \\
& a_{n, m_{j}, q} \geq 0, \quad \forall j \in\{1,2\}, \forall D_{m} \in \mathcal{D}_{q}, \forall C_{n} \in \mathcal{C}_{q}, \forall B S_{q} \in \mathcal{B} \\
& \mathcal{I}_{n, m, q} \in\{0,1\}, \quad \forall D_{m} \in \mathcal{D}_{q}, \forall C_{n} \in \mathcal{C}_{q}, \forall B S_{q} \in \mathcal{B}
\end{aligned}
$$

where

$$
\begin{aligned}
& \gamma_{U, B S} \triangleq\left(\gamma_{1,1}, \ldots, \gamma_{n, q}, \ldots, \gamma_{N, Q}\right) \\
& \gamma_{U, D R_{m_{r_{1}}}, B S} \triangleq\left(\gamma_{1, m_{r_{1}}, 1} \cdot \mathcal{I}_{1, m, 1}, \ldots, \gamma_{n, m_{r_{1}}, q} \cdot \mathcal{I}_{n, m, q}, \ldots, \gamma_{N, m_{r_{1}}, Q} \cdot \mathcal{I}_{N, m, Q}\right) \text {, }
\end{aligned}
$$

and

$$
\gamma_{U, D R_{m_{2}}, B S} \triangleq\left(\gamma_{1, m_{r_{2}}, 1} \cdot \mathcal{I}_{1, m, 1}, \ldots, \gamma_{n, m_{r_{2}}, q} \cdot \mathcal{I}_{n, m, q}, \ldots, \gamma_{N, m_{r_{2}}, Q} \cdot \mathcal{I}_{N, m, Q}\right)
$$

In problem J-GA-CA-PA, constraint (10a) enforces the total transmit power per channel, while constraint (10b) ensures that the total number of D2D groups assigned per user channel $C_{n} \in \mathcal{C}_{q}$ $\left(U_{n} \in \mathcal{U}_{q}\right)$ does not exceed one. Constraint (10c) ensures that the total number of D2D groups associated with each base-station $B S_{q}$ does not exceed $\xi_{q}$, while constraint (10d) ensures that no D2D group is paired to more than one user channel. Constraint (10e) ensures if D2D group $D_{m}$ is paired with user $U_{n}$ over channel $C_{n}$, then the SIC decoding order of the D2D receivers is preserved. Constraint (10f) ensures that if a user is paired to a D2D group, then the target minimum SINR must be satisfied. Constraint $(10 \mathrm{~g})$ ensures that the target minimum SINR for each $\mathrm{D} 2 \mathrm{D}$ receiver is satisfied if the $\mathrm{D} 2 \mathrm{D}$ group $D_{m}$ is paired to user $U_{n}$ (i.e. $\mathcal{I}_{n, m, q}=1$ ). Constraint (10h) ensures that the sum of power allocation coefficients of the D2D receivers does not exceed one if the D2D group $D_{m}$ is paired to user $U_{n}$ over channel $C_{n}$ (i.e. $\mathcal{I}_{n, m, q}=1$ ), and zero otherwise. The last four constraints define the range of values the decision variables take ${ }^{3}$.

Remark 4: Problem J-GA-CA-PA is non-convex and NP-complete [23,24], and thus is computationally-expensive. Moreover, maximizing the SINR of user may degrade the SINR of the D2D receivers (and vice versa). However, the minimum SINR constraints ensure sufficient QoS for all paired users and D2D groups. Nevertheless, the solution of problem J-GA-CA-PA is Pareto-optimal [25].

\footnotetext{
${ }^{3}$ In constraint (10i), if $\mathcal{I}_{n, m, q}=1$, then $0 \leq P_{n, m, q} \leq P$; otherwise $P_{n, m, q}=0$. That is, if a D2D group $D_{m} \in \mathcal{D}_{q}$ is not assigned a user channel $C_{n} \in \mathcal{C}_{q}$, then it is not allocated any transmit power.
} 
Based on Remark 4, the problem of joint D2D group association, channel assignment and power allocation is decoupled into two sub-problems: (1) multi-objective power allocation per (D2D group, user) pair, and (2) many-to-one stable matching.

\section{Multi-Objective SinR-Maximizing Power Allocation Per (D2D Group, USER) PAIR}

In this section, the aim is to determine the optimal multi-objective SINR-maximizing power allocation for each D2D group $D_{m} \in \mathcal{D}_{q}$ when paired with a certain user $U_{n} \in \mathcal{U}_{q}$ over channel $C_{n} \in \mathcal{C}_{q}$ within each cell/base-station $B S_{q} \in \mathcal{B}$. In particular, let the multi-objective SINRmaximizing power allocation (MO-SINR-MAX-PA) be defined as ${ }^{4}$

\section{MO-SINR-MAX-PA:}

$$
\begin{array}{ll}
\max & \left(\gamma_{n, q},\left\{\gamma_{n, m_{r_{1}}, q}\right\}_{D_{m} \in \mathcal{D}_{q}},\left\{\gamma_{n, m_{r_{2}}, q}\right\}_{D_{m} \in \mathcal{D}_{q}}\right) \\
\text { s.t. } & P_{n, q}+\sum_{D_{m} \in \mathcal{D}_{q}} \mathcal{I}_{n, m, q} P_{n, m, q} \leq P, \\
& \sum_{D_{m} \in \mathcal{D}_{q}} \mathcal{I}_{n, m, q} \leq 1, \\
& S_{n, m, q} \cdot \mathcal{I}_{n, m, q} \geq 0, \quad \forall D_{m} \in \mathcal{D}_{q}, \\
& \left(\gamma_{n, q}-\bar{\gamma}_{T_{U}}\right) \cdot \sum_{D_{m} \in \mathcal{D}_{q}} \mathcal{I}_{n, m, q} \geq 0, \\
& \left(\gamma_{n, m_{r_{j}}, q}-\bar{\gamma}_{T_{D R}}\right) \cdot \mathcal{I}_{n, m, q} \geq 0, \quad \forall j \in\{1,2\}, \forall D_{m} \in \mathcal{D}_{q}, \\
& a_{n, m_{r_{1}}, q}+a_{n, m_{r_{2}}, q} \leq \mathcal{I}_{n, m, q}, \quad \forall D_{m} \in \mathcal{D}_{q}, \\
& 0 \leq P_{n, m, q} \leq P \cdot \mathcal{I}_{n, m, q}, \quad \forall D_{m} \in \mathcal{D}_{q}, \\
& P_{n, q} \geq 0, \\
& a_{n, m_{r_{j}}, q} \geq 0, \quad \forall j \in\{1,2\}, \forall D_{m} \in \mathcal{D}_{q}, \\
& \mathcal{I}_{n, m, q} \in\{0,1\}, \quad \forall D_{m} \in \mathcal{D}_{q},
\end{array}
$$

where the constraints are as in problem J-GA-CA-PA; however, limited to each user $U_{n} \in \mathcal{U}_{q}$ within each base-station $B S_{q}$.

Remark 5: Problem MO-SINR-MAX-PA is still non-convex and NP-complete [23,24].

\footnotetext{
${ }^{4}$ The constraint on the maximum number of D2D groups that can be associated with a base-station is eliminated for now, as it will be incorporated later in the proposed stable matching algorithms.
} 
Alternatively, problem MO-SINR-MAX-PA can be solved efficiently for each (D2D group, user) pair. To that end, let $\boldsymbol{\Phi}_{n, q}=\left\{\boldsymbol{\Phi}_{n, 1, q}, \ldots, \boldsymbol{\Phi}_{n, m, q}, \ldots, \boldsymbol{\Phi}_{n,\left|\mathcal{D}_{q}\right|, q}\right\}$ be the set of all possible combinations of pairing a D2D group $D_{m} \in \mathcal{D}_{q}$ with user $U_{n} \in \mathcal{U}_{q}$, where $\left|\Phi_{n, q}\right|=\left|\mathcal{D}_{q}\right|$. Hence, for each combination $\boldsymbol{\Phi}_{n, m, q} \in \boldsymbol{\Phi}_{n, q}$, problem MO-SINR-MAX-PA is reformulated as ${ }^{5}$

R-MO-SINR-MAX-PA $\left(\boldsymbol{\Phi}_{n, m, q}\right)$ :

$$
\begin{array}{ll}
\max & \left(\gamma_{n, q}^{m}, \gamma_{n, m_{r_{1}}, q}, \gamma_{n, m_{r_{2}}, q}\right) \\
\text { s.t. } & P_{n, q}^{m}+P_{n, m, q} \leq P \\
& S_{n, m, q} \geq 0 \\
& \gamma_{n, q}^{m} \geq \bar{\gamma}_{T_{U}}, \\
& \gamma_{n, m_{r_{j}}, q} \geq \bar{\gamma}_{T_{D R}}, \quad \forall j \in\{1,2\}, \\
& a_{n, m_{r_{1}}, q}+a_{n, m_{r_{2}}, q} \leq 1, \\
& 0 \leq P_{n, m, q} \leq P, \\
& P_{n, q}^{m} \geq 0, \\
& a_{n, m_{r_{j}}, q} \geq 0, \quad \forall j \in\{1,2\},
\end{array}
$$

where $P_{n, q}^{m}$ is the transmit power of user $U_{n} \in \mathcal{U}_{q}$ when paired with D2D group $D_{m} \in \mathcal{D}_{q}$, while $\gamma_{n, q}^{m}$ is the corresponding SINR.

Remark 6: The SINR function $\gamma_{n, q}^{m}$ can be verified to be a linear-fractional (LF) function in $P_{n, q}^{m}, \forall U_{n} \in \mathcal{U}_{q}$. However, the SINR functions $\gamma_{n, m_{r_{j}}, q}$ (for $j \in\{1,2\}$ ) (in (7) and (8)) are non-linear fractional functions in $P_{n, m, q}$ and $a_{n, m_{j}, q}, \forall D_{m} \in \mathcal{D}_{q}, \forall B S_{q} \in \mathcal{B}$.

To linearize problem R-MO-SINR-MAX-PA $\left(\Phi_{n, m, q}\right)$, define $\bar{P}_{n, m_{r_{j}}, q} \triangleq P_{n, m, q} a_{n, m_{r_{j}}, q}$, for $\forall j \in\{1,2\}$, where it should be noted that $0 \leq \bar{P}_{n, m_{r_{j}}, q} \leq P_{n, m, q}, \forall j \in\{1,2\}$. Additionally, since $a_{n, m_{r_{1}}, q}+a_{n, m_{r_{2}}, q} \leq 1$, then $\bar{P}_{n, m_{r_{1}}, q}+\bar{P}_{n, m_{r_{2}}, q} \leq P_{n, m, q}$. Consequently and based on (4), (7) and (8), constraints (15c) and (15d) can be re-expressed as

$$
\begin{gathered}
P_{n, q}^{m}\left|h_{n, q}\right|^{2} \geq \bar{\gamma}_{T_{U}}\left(P_{n, m, q}\left|h_{n, m, q}\right|^{2}+N_{0}\right), \\
\left|f_{n, m_{t}, m_{r_{2}}, q}\right|^{2} \bar{P}_{n, m_{r_{2}}, q} \geq \bar{\gamma}_{T_{D R}}\left(\left|f_{n, m_{t}, m_{r_{2}}, q}\right|^{2} \bar{P}_{n, m_{r_{1}}, q}+P_{n, q}^{m}\left|z_{n, m_{r_{2}}, q}\right|^{2}+N_{0}\right),
\end{gathered}
$$

and

$$
\left|f_{n, m_{t}, m_{r_{1}}, q}\right|^{2} \bar{P}_{n, m_{r_{1}}, q} \geq \bar{\gamma}_{T_{D R}}\left(P_{n, q}^{m}\left|z_{n, m_{r_{1}}, q}\right|^{2}+N_{0}\right)
$$

respectively. Therefore, problem R-MO-SINR-MAX-PA $\left(\boldsymbol{\Phi}_{n, m, q}\right)$ is re-written as

\footnotetext{
${ }^{5}$ All binary decision variables are eliminated from $\gamma_{n, q}$, since a specific combination of (D2D group, user) pair is considered.
} 


\section{R-MO-SINR-MAX-PA $\left(\Phi_{n, m, q}\right)$ :}

$$
\begin{array}{ll}
\max & \left(\gamma_{n, q}^{m}, \gamma_{n, m_{r_{1}}, q}, \gamma_{n, m_{r_{2}}, q}\right) \\
\text { s.t. } & P_{n, q}^{m}+P_{n, m, q} \leq P, \\
& S_{n, m, q} \geq 0, \\
& P_{n, q}^{m}\left|h_{n, q}\right|^{2} \geq \bar{\gamma}_{T_{U}}\left(P_{n, m, q}\left|h_{n, m, q}\right|^{2}+N_{0}\right), \\
& \left|f_{n, m_{t}, m_{r_{1}}, q}\right|^{2} \bar{P}_{n, m_{r_{1}}, q} \geq \bar{\gamma}_{T_{D R}}\left(P_{n, q}^{m}\left|z_{n, m_{r_{1}}, q}\right|^{2}+N_{0}\right), \\
& \left|f_{n, m_{t}, m_{r_{2}}, q}\right|^{2} \bar{P}_{n, m_{r_{2}}, q} \geq \bar{\gamma}_{T_{D R}}\left(\left|f_{n, m_{t}, m_{r_{2}}, q}\right|^{2} \bar{P}_{n, m_{r_{1}}, q}+P_{n, q}^{m}\left|z_{n, m_{r_{2}}, q}\right|^{2}+N_{0}\right), \\
& \bar{P}_{n, m_{r_{1}}, q}+\bar{P}_{n, m_{r_{2}}, q} \leq P_{n, m, q}, \\
& 0 \leq P_{n, m, q} \leq P, \\
& P_{n, q}^{m} \geq 0,
\end{array}
$$

where all the constraints are linear, and all SINR functions are LF functions, which also happen to be pseudo-linear functions (i.e. both pseudo-convex and pseudo-concave) [26,27].

Problem R-MO-SINR-MAX-PA $\left(\boldsymbol{\Phi}_{n, m, q}\right)$ can now be solved via a parametric approach [28,29]. Particularly, the SINR function $\gamma_{n, q}^{m}$ of each user $U_{n} \in \mathcal{U}_{q}$ attains its maximum when the transmit power of the D2D group $D_{m} \in \mathcal{D}_{q}$ is nulled (i.e. $P_{n, m, q}=0$, and $P_{n, q}^{m}=P$ ), as

$$
\bar{\gamma}_{n, q}^{m} \triangleq \frac{P\left|h_{n, q}\right|^{2}}{N_{0}} .
$$

Similarly, the SINR $\gamma_{n, m_{r_{2}}, q}$ for $D R_{m_{2}}$ is maximized when $P_{n, q}^{m}=0$, and $\bar{P}_{n, m_{r_{1}}, q}=0$, yielding

$$
\bar{\gamma}_{n, m_{r_{2}}, q} \triangleq \frac{P\left|f_{n, m_{t}, m_{r_{2}}, q}\right|^{2}}{N_{0}} .
$$

Lastly, the SINR of $D R_{m_{1}}$ is maximized as

$$
\bar{\gamma}_{n, m_{r_{1}}, q} \triangleq \frac{P\left|f_{n, m_{t}, m_{r_{1}}, q}\right|^{2}}{N_{0}} .
$$

Consequently, the maximized SINR values $\bar{\gamma}_{n, q}^{m}$ and $\bar{\gamma}_{n, m_{r_{j}}, q}$ (for $j \in\{1,2\}$ ) can be expressed in parametric form as [29]

$$
\begin{gathered}
\Gamma_{n, q}^{m} \triangleq P_{n, q}^{m}\left|h_{n, q}\right|^{2}-\bar{\gamma}_{n, q}^{m}\left(P_{n, m, q}\left|h_{n, m, q}\right|^{2}+N_{0}\right), \\
\Gamma_{n, m_{r_{2}}, q} \triangleq\left|f_{n, m_{t}, m_{r_{2}}, q}\right|^{2} \bar{P}_{n, m_{r_{2}}, q}-\bar{\gamma}_{n, m_{r_{2}}, q}\left(\left|f_{n, m_{t}, m_{r_{2}}, q}\right|^{2} \bar{P}_{n, m_{r_{1}}, q}+P_{n, q}^{m}\left|z_{n, m_{r_{2}}, q}\right|^{2}+N_{0}\right),
\end{gathered}
$$

and

$$
\Gamma_{n, m_{r_{1}}, q} \triangleq\left|f_{n, m_{t}, m_{r_{1}}, q}\right|^{2} \bar{P}_{n, m_{r_{1}}, q}-\bar{\gamma}_{n, m_{r_{1}}, q}\left(P_{n, q}^{m}\left|z_{n, m_{r_{1}}, q}\right|^{2}+N_{0}\right) \text {, }
$$

respectively. Thus, problem R-MO-SINR-MAX-PA $\left(\boldsymbol{\Phi}_{n, m, q}\right)$ is transformed into its parametric form as $[28,30]$ 


\section{P-R-MO-SINR-MAX-PA $\left(\Phi_{n, m, q}\right)$ :}

$\max \Gamma_{n, q}^{m}+\left(\Gamma_{n, m_{r_{1}}, q}+\Gamma_{n, m_{r_{2}}, q}\right)$

s.t. Constraints (19a) - (19h),

which can be verified to be a linear programming (LP) problem. Therefore, it can be efficiently solved via any standard optimization package [31].

Lemma 1: The optimal solution to problem P-R-MO-SINR-MAX-PA $\left(\Phi_{n, m, q}\right)$ for each $\boldsymbol{\Phi}_{n, m, q} \in \boldsymbol{\Phi}_{n, q}$ is the global optimal solution to problem R-MO-SINR-MAX-PA $\left(\boldsymbol{\Phi}_{n, m, q}\right)$.

Proof: The proof in [30] is tenable to our work.

To determine the SINR of each D2D group $D_{m} \in \mathcal{D}_{q}$ and user $U_{n} \in \mathcal{U}_{q}$ when paired over a channel $C_{n} \in \mathcal{C}_{q}$, the following solution procedure is devised. Specifically, the goal is to iterate over all possible combinations in $\boldsymbol{\Phi}_{n, q}$, and solve problem P-R-MO-SINR-MAX-PA $\left(\boldsymbol{\Phi}_{n, m, q}\right)$ for each combination $\boldsymbol{\Phi}_{n, m, q} \in \boldsymbol{\Phi}_{n, q}$ within each base-station $B S_{q} \in \mathcal{B}$. Upon convergence of the solution procedure, each D2D group $D_{m}$ determines the sum of SINR values of its receivers when paired with user $U_{n} \in \mathcal{U}_{q}$, as

$$
\Upsilon_{n, m, q} \triangleq \mathcal{J}_{n, m, q} \cdot\left(\gamma_{n, m_{r_{1}}, q}+\gamma_{n, m_{r_{2}}, q}\right),
$$

where $\mathcal{J}_{n, m, q}$ is a binary indicator function set as $\mathcal{J}_{n, m, q}=1$ if problem P-R-MO-SINR-MAXPA $\left(\Phi_{n, m, q}\right)$ is successfully solved and all constraints are satisfied, and $\mathcal{J}_{n, m, q}=0$ otherwise. More specifically, if user $U_{n}$ or any D2D receiver $D R_{m_{j}}$ (for $j \in\{1,2\}$ ) cannot meet the target minimum SINR, then $\mathcal{J}_{n, m, q}=0$. On the other hand, for each base-station $B S_{q} \in \mathcal{B}$, the sum of SINR values resulting from pairing user $U_{n} \in \mathcal{U}_{q}$ with D2D group $D_{m} \in \mathcal{D}_{q}$ is determined as

$$
\Psi_{n, m, q} \triangleq \mathcal{J}_{n, m, q} \cdot\left(\gamma_{n, q}^{m}+\gamma_{n, m_{r_{1}, q}}+\gamma_{n, m_{r_{2}}, q}\right) .
$$

The proposed solution procedure for multi-objective SINR-maximizing power allocation (SPMO-SINR-MAX-PA) per (D2D group, user) pair of each base-station $B S_{q} \in \mathcal{B}$ is given in Algorithm 1. Finally, the obtained $\Upsilon_{n, m, q}$ and $\Psi_{n, m, q}$ values $\forall U_{n} \in \mathcal{U}_{q}, \forall D_{m} \in \mathcal{D}_{q}$ and $\forall B S_{q} \in \mathcal{B}$ are used to determine the preference lists of the D2D groups and base-stations used in the proposed stable matching algorithms to obtain stable matching solutions with preferences over (D2D group, user) pairs.

Remark 7: If no D2D group is paired with user $U_{n} \in \mathcal{U}_{q}$ over channel $C_{n} \in \mathcal{C}_{q}$, then $P_{n, q}=P$ and hence $\gamma_{n, q}=P\left|h_{n, q}\right|^{2} / N_{0}$. 


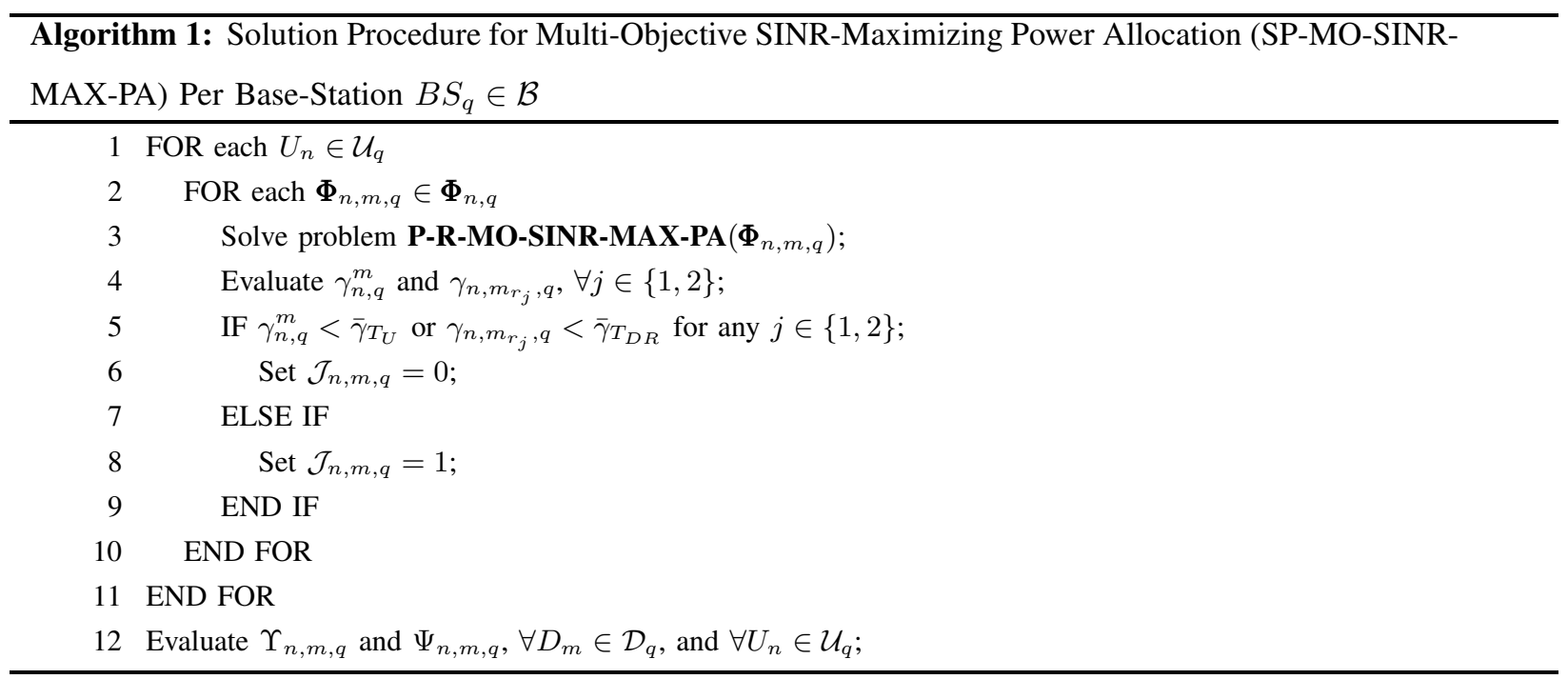

Remark 8: If $\mathcal{J}_{n, m, q}=0$ (i.e. $\Upsilon_{n, m, q}=0$ ) for any D2D group $D_{m} \in \mathcal{D}_{q}$, then user $U_{n} \in \mathcal{U}_{q}$ is considered unacceptable to that D2D group. In a similar manner, if $\Psi_{n, m, q}=0$, then the pair $\left(D_{m}, U_{n}\right)$ is considered unacceptable to base-station $B S_{q} \in \mathcal{B}$.

Remark 9: The greater the value of $\Upsilon_{n, m, q}$ is, the more preferred is user $U_{n} \in \mathcal{U}_{q}$ to D2D group $D_{m} \in \mathcal{D}_{q}$. In a similar manner, the greater the value of $\Psi_{n, m, q}$ is, the more preferred is the pair $\left(D_{m}, U_{n}\right)$ to base-station $B S_{q} \in \mathcal{B}$.

Remark 10: The SP-MO-SINR-MAX-PA can be executed locally at each base-station $B S_{q} \in$ $\mathcal{B}$, and involves at total of $\left|\mathcal{U}_{q}\right| \cdot\left|\mathcal{D}_{q}\right|$ iterations, in each of which a LP problem is efficiently solved. Therefore, SP-MO-SINR-MAX-PA can be executed with minimal computational complexity.

Remark 11: The proposed solution procedure eliminates the need for swap-operations employed in $[13,16,17]$, which were used to ensure stability after executing the matching algorithm and power allocation, due to the resulting inter-user interference and possible violation of the QoS constraints. Specifically, the SP-MO-SINR-MAX-PA determines the optimal power allocation per (D2D group, user) pair, and simultaneously takes into account the interference at the cellular user as well as the D2D receivers, while maintaining QoS requirements. That is, the preference lists are based on optimized power allocation and contain only acceptable users and (D2D group, user) pairs. This in turn significantly reduces signaling and communication overheads as well as computational complexity. 


\section{Stable Matching with Preferences over (D2D Group, User) Pairs}

In the classical student-project allocation (SPA) matching problem, the aim is to associate students with lecturers, who offer projects to students. Each lecturer has a quota representing the maximum number of students he/she can supervise, whereas each project offered by each lecturer can be assigned to one or more students [18]. Also, each lecturer has preferences over the potential students, while each student has preferences over the offered projects. Consequently, and based on the students' and lecturers' preferences, the goal is to find a stable matching of students and projects offered by each lecturer, while satisfying the lecturers and projects quotas. In many instances, a lecturer may prefer a student to work on a certain project. In turn, in this work, a modified version of the SPA matching problem is considered-namely, SPA with preferences over (student, project) pairs—where each student has preferences over the available projects, while each lecturer has preferences over (student, project) pairs [19]. By analogy, the students represent the D2D groups, while the projects are the available cellular user channels, and the lecturers are the base-stations. More importantly, each D2D group has preferences over the acceptable cellular users, while each base-station has preferences over the acceptable (D2D group, user) pairs, and the aim is to associate D2D groups with users of each base-station, such that a stable matching is obtained.

An instance of SPA-(D2D group, user) consists of a set of D2D groups $\mathcal{D}$, a set of users $\mathcal{U}$, and a set of base-stations $\mathcal{B}$. More formally, the following definitions are required.

\section{A. Definitions}

Definition 1 (Acceptability): A user $U_{n} \in \mathcal{U}_{q}$ is considered acceptable to D2D group $D_{m} \in \mathcal{D}_{q}$ if $\Upsilon_{n, m, q}>0$. Also, a (D2D group, user) pair $\left(D_{m}, U_{n}\right)$ is said to be acceptable to base-station $B S_{q}$ if $\Psi_{n, m, q}>0$. In turn, let $\mathcal{A}_{D_{m}}$ be the list of acceptable users by D2D group $D_{m} \in \mathcal{D}_{q}$. Similarly, let $\mathcal{A}_{B S_{q}}$ be the list of acceptable (D2D group, user) pairs to base-station $B S_{q}$.

Definition 2 (Assignment): Let an assignment $\mathcal{M}$ be defined as a subset of $\mathcal{D} \times \mathcal{U}$, such that $\left(D_{m}, U_{n}\right) \in \mathcal{M}$, where user $U_{n} \in \mathcal{A}_{D_{m}}$ is acceptable to D2D group $D_{m}$, and the pair $\left(D_{m}, U_{n}\right) \in \mathcal{A}_{B S_{q}}$ is acceptable to some base-station $B S_{q} \in \mathcal{B}$. In other words, if $\left(D_{m}, U_{n}\right) \in \mathcal{M}$, then D2D group $D_{m}$ is said to be paired with user $U_{n}$ and vice versa. For notational convenience, let $\mathcal{M}\left(D_{m}\right)=U_{n}$ denote that user $U_{n}$ is paired to $D_{m}$ in $\mathcal{M}$. Similarly, $\mathcal{M}\left(U_{n}\right)=D_{m}$ implies that $D_{m}$ is paired with $U_{n}$ in $\mathcal{M}$. Lastly, $\mathcal{M}\left(B S_{q}\right)=D_{m}$ implies that $D_{m}$ is associated with $B S_{q}$ in $\mathcal{M}$, and vice versa. 
Definition 3 (Preference): If D2D group $D_{m}$ prefers user $U_{n}$ to $U_{l}$ (i.e. $\Upsilon_{n, m, q}>\Upsilon_{l, m, q}$ for $n \neq l)$, then $U_{n} \succ_{D_{m}} U_{l}$. Similarly, if base-station $B S_{q}$ prefers the pair $\left(D_{m}, U_{n}\right)$ to $\left(D_{w}, U_{l}\right)$ (i.e. $\Psi_{n, m, q}>\Psi_{l, w, q}$ for $n \neq l$ and $\left.m \neq w\right)$, then $\left(D_{m}, U_{n}\right) \succ_{B S_{q}}\left(D_{w}, U_{l}\right)$.

Definition 4 (Preference List): Let $\mathbb{P}_{D_{m}}=\left\{U_{n}^{(1)}, \ldots, U_{l}^{\left(\left|\mathcal{A}_{D_{m}}\right|\right)}\right\}$ be the preference list of D2D group $D_{m}$, where $U_{n}^{(1)}\left(U_{l}^{\left(\left|\mathcal{A}_{D_{m}}\right|\right)}\right)$ indicates that $U_{n}\left(U_{l}\right)$ is the most (least) preferred user to D2D group $D_{m}$. Similarly, $\mathbb{P}_{B S_{q}}=\left\{\left(D_{m}, U_{n}\right)^{(1)}, \ldots,\left(D_{w}, U_{l}\right)^{\left|\mathcal{A}_{B S_{q}}\right|}\right\}$, where $\left(D_{m}, U_{n}\right)^{(1)}$ $\left(\left(D_{w}, U_{l}\right)^{\left|\mathcal{A}_{B S_{q}}\right|}\right)$ indicates that the pair $\left(D_{m}, U_{n}\right)\left(\left(D_{w}, U_{l}\right)\right)$ is the most (least) preferred by base-station $B S_{q} \in \mathcal{B}$.

Definition 5 (Projected Preference List): The projected preference list $\overline{\mathbb{P}}_{B S_{q}}^{n}$ of base-station $B S_{q} \in \mathcal{B}$ is obtained from $\mathbb{P}_{B S_{q}}$ by deleting all the pairs which do not find $U_{n} \in \mathcal{U}_{q}$ acceptable.

Definition 6 (Subscription): Any $U_{n} \in \mathcal{U}_{q}$ is said to be under-subscribed, full, or oversubscribed if $\left|\mathcal{M}\left(U_{n}\right)\right|$ is less than, equal to, or greater than one, respectively. Also, $B S_{q} \in \mathcal{B}$ is said to be under-subscribed, full, or over-subscribed, if $\left|\mathcal{M}\left(B S_{q}\right)\right|$ is less, equal to, or greater than $\xi_{q}$, respectively.

Definition 7 (Matching): An assignment $\mathcal{M} \subset \mathcal{D} \times \mathcal{U}$ is called a matching if:

(a) For each user $U_{n} \in \mathcal{U},\left|\mathcal{M}\left(U_{n}\right)\right| \leq 1$.

(b) For each D2D group $D_{m} \in \mathcal{D},\left|\mathcal{M}\left(D_{m}\right)\right| \leq 1$.

(c) For each base-station $B S_{q} \in \mathcal{B},\left|\mathcal{M}\left(B S_{q}\right)\right| \leq \xi_{q}$.

That is, each base-station $B S_{q} \in \mathcal{B}$ is associated with at most $\xi_{q}$ D2D groups in matching $\mathcal{M}$, while each user $U_{n} \in \mathcal{U}$ can be paired with at most one D2D group $D_{m} \in \mathcal{D}$, and vice versa.

Definition 8 (Blocking Pair): The pair $\left(D_{m}, U_{n}\right) \in \mathcal{D} \times \mathcal{U} \backslash \mathcal{M}$ is said to block a matching $\mathcal{M}$ if [19]:

(a) $U_{n} \in \mathcal{A}_{D_{m}}$ (i.e. user $U_{n}$ is acceptable to D2D group $D_{m}$ ).

(b) Either $D_{m}$ is unmatched in $\mathcal{M}$ or $D_{m}$ prefers $U_{n} \in \mathcal{U}_{q}$ to $\mathcal{M}\left(D_{m}\right)$.

(c) Either:

(c.1) $U_{n}$ is under-subscribed, and either:

(c.1.1) $\mathcal{M}\left(D_{m}\right) \in \mathcal{U}_{q}$, and $B S_{q}$ prefers $\left(D_{m}, U_{n}\right)$ to $\left(D_{m}, \mathcal{M}\left(D_{m}\right)\right)$, or

(c.1.2) $\mathcal{M}\left(D_{m}\right) \notin \mathcal{U}_{q}$, and $B S_{q}$ is under-subscribed, or

(c.1.3) $\mathcal{M}\left(D_{m}\right) \notin \mathcal{U}_{q}, B S_{q}$ is full, and $B S_{q}$ prefers $\left(D_{m}, U_{n}\right)$ to the worst pair $\left(D_{w}, U_{l}\right)$ that is associated with $B S_{q}$, or

(c.2) $U_{n}$ is full, and $B S_{q}$ prefers $\left(D_{m}, U_{n}\right)$ to $\left(D_{w}, U_{n}\right)$, with $D_{w}$ being the worst D2D group in $\mathcal{M}\left(U_{n}\right)$, and either: 
(c.2.1) $\mathcal{M}\left(D_{m}\right) \notin \mathcal{U}_{q}$, or

(c.2.2) $\mathcal{M}\left(D_{m}\right) \in \mathcal{U}_{q}$, and $B S_{q}$ prefers $\left(D_{m}, U_{n}\right)$ to $\left(D_{m}, \mathcal{M}\left(D_{m}\right)\right)$.

Definition 9 (Stable Matching): A matching $\mathcal{M}$ is said to be stable if it admits no blocking pair.

\section{B. Algorithms Description}

This subsection describes the D2D groups-oriented stable matching (DG-SM), and basestations-oriented stable matching (BS-SM) algorithms ${ }^{6}$, which are adapted from $[18,19]$.

1) D2D Groups-Oriented Stable Matching: In the DG-SM algorithm, all D2D groups are initially set to be free, and all the users and base-stations are assumed to be completely unsubscribed. In each iteration, a free D2D group is assigned to the first preferred user on its preference list. This results in a temporary assignment between D2D groups, users and base-stations, which may be broken later when a user or base-station becomes over-subscribed. Additionally, some entries in the preference lists of D2D groups and base-stations may be deleted when a user or a base-station becomes full. In particular, deleting a pair $\left(D_{m}, U_{n}\right)$ during the execution of the DG-SM algorithm constitutes deleting user $U_{n}$ from the preference list of D2D group $D_{m}$, and deleting the pair $\left(D_{m}, U_{n}\right)$ from the preference list of base-station $B S_{q}$. Moreover, if any user $U_{n} \in \mathcal{U}_{q}$ becomes full during the execution of the DG-SM algorithm, then it may only become under-subscribed again if base-station $B S_{q}$ becomes over-subscribed, and one of its assignments with $U_{n}$ is broken [19]. Furthermore, if a base-station $B S_{q}$ becomes full during the execution of the algorithm, then it never becomes under-subscribed again. The DG-SM algorithm is outlined in Algorithm 2.

2) Base-Stations-Oriented Stable Matching: As in the DG-SM algorithm, the BS-SM algorithm starts initially by setting all D2D groups free, while assuming all users and base-stations to be completely unsubscribed. Then, the algorithm finds the first unpaired D2D group/user $\left(D_{m}, U_{n}\right)$, where $U_{n}$ and its associated base-station $B S_{q}$ are both under-subscribed. If such pair is found and $D_{m}$ is already paired with another user $U_{l}$, the pair $\left(D_{m}, U_{l}\right)$ is first broken before the new pair $\left(D_{m}, U_{n}\right)$ is assigned, and all pairs inferior to the pair $\left(D_{m}, U_{l}\right)$ are deleted from $B S_{q}$ 's preference list. This process repeats until convergence to the $\mathcal{M}_{B S}$ stable matching solution. The BS-SM algorithm is listed in Algorithm 3.

\footnotetext{
${ }^{6}$ The DG-SM and BS-SM algorithms can be executed efficiency among base-stations via backhaul links [32,33], without the need of a centralized controller.
} 

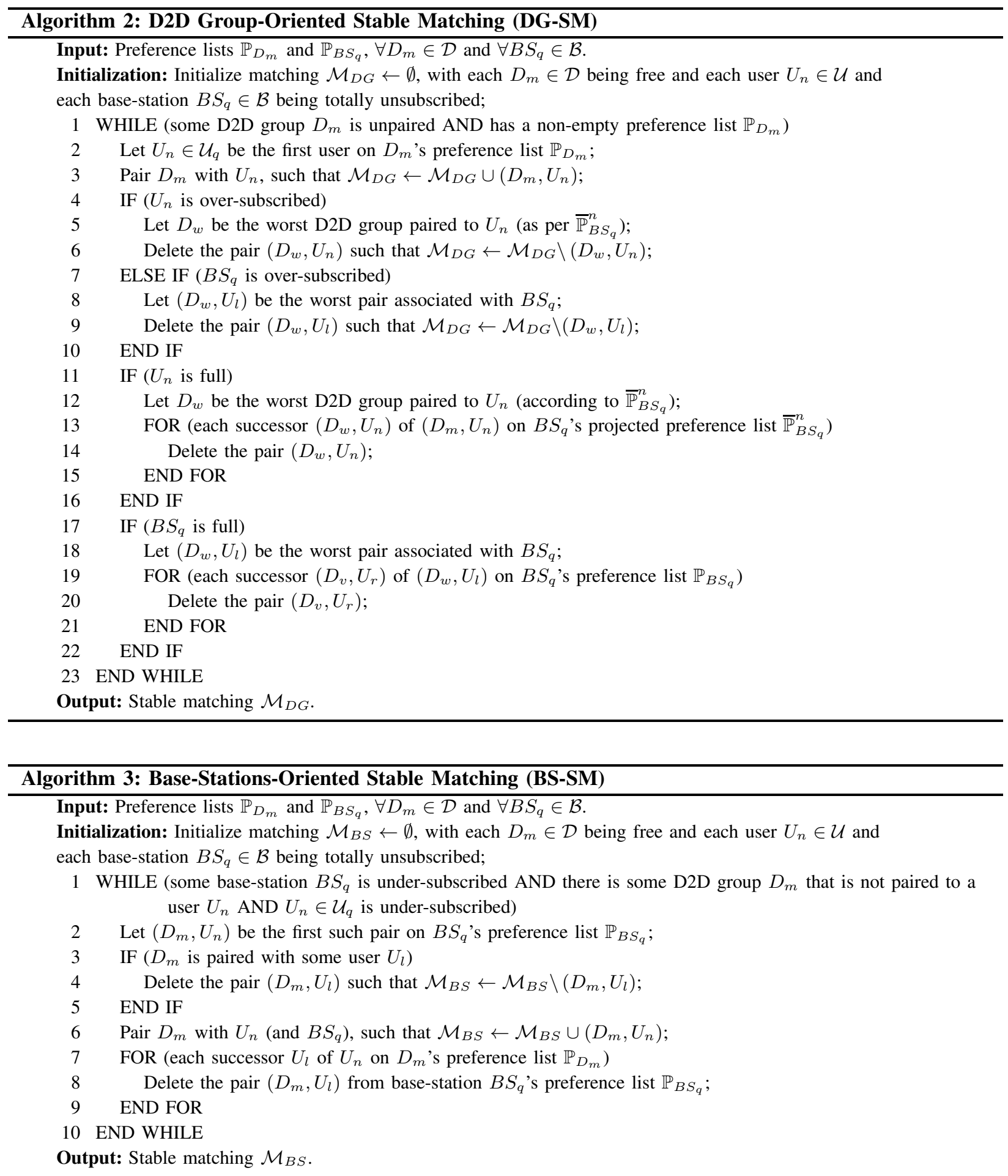

\section{Properties}

\section{1) Convergence to a Stable Matching Solution:}

Lemma 2: The DG-SM and BS-SM algorithms converge in a finite number of iterations to a stable matching. 
Proof: The proof is approached by showing that: (i) any execution of any of the algorithms terminates with a matching in a finite number of iterations, (ii) no blocking pair is deleted during an algorithm execution, and (iii) the algorithm execution admits no blocking pair, and hence the generated matching is stable [18].

In DG-SM, condition (i) holds since in each iteration a free D2D group applies to its most preferred user. Also, since no D2D group can apply to the same user twice, due to the deletion of corresponding (D2D group, user) pair, a matching is guaranteed to emerge after a number of iterations, limited by the aggregate D2D group preference lists. Condition (ii) is proved by showing that a deleted pair, during the algorithm execution, can neither satisfy (c.1) nor (c.2) of the blocking pair definition (i.e. Definition 8). Finally, condition (iii) is established from the fact that any blocking pair of the generated matching either contradicts with condition (i) if it is deleted, or contradicts with condition (ii) otherwise. Hence, a blocking pair is never admitted by any arbitrary execution of the algorithm [19].

In BS-SM, condition (i) holds because in each iteration, a D2D group is either provisionally assigned to a new user, or to a better user than the currently assigned one until the loop condition is never met; whereas condition (ii) is guaranteed since any blocking pair deleted during an execution must contradict with (b) in Definition 8. Lastly, the algorithm's stability (i.e. condition (iii)) is proved by contradiction as follows. Assuming a (D2D group, user) pair blocking the matching generated by an arbitrary execution as guaranteed by condition (i), this pair is not deleted according to (ii). However, this can be shown to contradict with both of (c.1) and (c.2) of Definition 8, and this completes the proof.

\section{2) Complexity:}

Lemma 3: The DG-SM and BS-SM algorithms have polynomial-time complexity of $\mathcal{O}(|\mathcal{U}| \cdot|\mathcal{D}|)$, where $|\mathcal{U}|$ and $|\mathcal{D}|$ are the total number of users and D2D groups in the network, respectively.

Proof: It can be easily verified that in the worst-case scenario of the DG-SM algorithm, each free D2D group $D_{m} \in \mathcal{D}$ with a non-empty preference list applies to at least one user in the user set $\mathcal{U}$. Additionally, during the execution of the algorithm, some (D2D group, user) pairs may be deleted and their entries in the preference lists of the corresponding D2D groups and base-stations are deleted. Consequently, the worst-case complexity of the DG-SM algorithm is $\mathcal{O}(|\mathcal{U}| \cdot|\mathcal{D}|)$. A similar argument also applies to the BS-SM algorithm.

In fact, the complexity of both algorithms is much lower than $\mathcal{O}(|\mathcal{U}| \cdot|\mathcal{D}|)$, since $\mathrm{D} 2 \mathrm{D}$ groups can only apply (and potentially be paired) to users within the cell it falls into, and each base- 
station can only associate D2D groups and pair them to users within its cell.

\section{3) Optimality:}

Lemma 4: The stable matching obtained via the DG-SM (BS-SM) algorithm is optimal with respect to each paired D2D group (base-station).

Proof: The optimality of the stable matching achieved by DG-SM-in the sense that D2D groups obtain their best matching compared to any other stable matching, and that any unassigned group is unassigned in any stable matching — can be shown to hold as follows. First, the fact that no stable pair is deleted during an execution of the algorithm must be established, where a stable pair is one that is part of some stable matching [18]. This property is proved by showing that the first stable pair $\left(D_{m}, U_{n}\right)$ deleted due to $U_{n}$ becoming full, entails the existence of a more preferred pair by $B S_{q}$ - the base station offering $U_{n}$ - than the worst D2D group in any matching $\mathcal{M}_{D G}^{\prime}$ that involves $\left(D_{m}, U_{n}\right)$. Since such matching is always blocked, this contradicts the fact that the deleted pair was a stable pair. Likewise, when $\left(D_{m}, U_{n}\right)$ is blocked because $B S_{q}$ becomes full, it can be shown that a pair must always exist to block $\mathcal{M}_{D G}^{\prime}$. Hence, a stable matching in which each D2D group is allocated its first preference, where no stable pair is deleted, is optimal.

On the other hand, the matching $\mathcal{M}_{B S}$ formed by BS-SM has a weaker optimality notion, whereby each base-station prefers its assigned pairs to those obtained in any other matching $\mathcal{M}_{B S}^{\prime}$ with a different set of allocated pairs. The proof is tackled by showing that a one-to-one mapping function $\mathcal{X}$ from $\widehat{\mathcal{M}}_{B S}\left(B S_{q}\right)=\mathcal{M}_{B S}^{\prime}\left(B S_{q}\right) \backslash \mathcal{M}_{B S}\left(B S_{q}\right)$ to $\widetilde{\mathcal{M}}_{B S}\left(B S_{q}\right)=\mathcal{M}_{B S}\left(B S_{q}\right) \backslash$ $\mathcal{M}_{B S}^{\prime}\left(B S_{q}\right)$ holds with the property that $B S_{q}$ prefers $\mathcal{X}((D, U))$ to $(D, U), \forall(D, U) \in \mathcal{D} \times \mathcal{U}$ [18]. The mapping is iteratively constructed by considering a pair $\left(D_{m}, U_{n}\right) \in \widehat{\mathcal{M}}_{B S}\left(B S_{q}\right)$ that is preferred by $B S_{q}$ to at least one pair in $\mathcal{M}_{B S}\left(B S_{q}\right)$. To avoid blocking $\mathcal{M}_{B S}, U_{n}$ must be fully-subscribed in $\mathcal{M}_{B S}$ with pairs whom $B S_{q}$ prefers to $\left(D_{m}, U_{n}\right)$. Thus, there must be a pair $\left(D_{w}, U_{n}\right) \notin \mathcal{M}_{B S}^{\prime}\left(B S_{q}\right)$ which is in turn mapped to $\left(D_{m}, U_{n}\right)$ in $\mathcal{X}$. After mapping all such pairs, all dominated pairs in $\widehat{\mathcal{M}}_{B S}\left(B S_{q}\right)$-which are worse than every pair in $\mathcal{M}_{B S}\left(B S_{q}\right)$ can be arbitrarily mapped to the remaining pairs in $\widetilde{\mathcal{M}}_{B S}\left(B S_{q}\right)$. Hence, $B S_{q}\left(\forall B S_{q} \in \mathcal{B}\right)$ must prefer $\mathcal{M}_{B S}$ to $\mathcal{M}_{B S}^{\prime}$, and this completes the proof.

Remark 12: The proposed stable matching algorithms ensure network stability. Particularly, stability refers to the case where no D2D group or base-station wishes to deviate and change its pairing/assocation. This in turn minimizes communications overheads and delays, and ensures stable network connectivity. 
Remark 13: The proposed stable matching algorithms pose a trade-off between optimality (in terms of resulting SINR per user and D2D receiver), complexity, and stability in comparison to the J-GA-CA-PA scheme. In other words, some of the global optimality is lost due to the enforced stability across all base-stations and (D2D group, user) pairs, but in return for much lower computational complexity. Last-but-not-least, the resulting D2D group association and channel assignment by the J-GA-CA-PA is not necessarily stable, as it strictly aims at maximizing the SINR of all users and D2D receivers, irrespective of their stability.

\section{Simulation Results}

This section evaluates the performance of the proposed DG-SM and BS-SM algorithms when combined with the solution procedure SP-MO-SINR-MAX-PA, and are respectively denoted SP-DG-SM and SP-BS-SM for short. The network is assumed to have $Q=3$ base-stations, $N=9$ cellular users, and $M=6$ D2D groups, located as illustrated in Fig. 2, where the D2D receivers are located within $2.5 \mathrm{~m}$ from each $\mathrm{D} 2 \mathrm{D}$ transmitter. The cellular user subsets associated with each base-station $B S_{q}$ (for $q \in\{1,2,3\}$ ) are $\mathcal{U}_{1}=\left\{U_{1}, U_{2}, U_{3}\right\}, \mathcal{U}_{2}=\left\{U_{4}, U_{5}, U_{6}\right\}$, and $\mathcal{U}_{3}=\left\{U_{7}, U_{8}, U_{9}\right\}$, respectively, where each user is allocated a dedicated channel. Additionally, the D2D groups that fall within the coverage area of each base-station are given by $\mathcal{D}_{1}=$ $\left\{D_{1}, D_{2}, D_{3}\right\}, \mathcal{D}_{2}=\left\{D_{3}, D_{4}, D_{5}\right\}$, and $\mathcal{D}_{3}=\left\{D_{2}, D_{3}, D_{5}, D_{6}\right\}$. Thus, the potential (D2D group, user) pairings and base-station associations ${ }^{7}$ are summarized in Table II.

TABLE II

Potential (D2D Group, USER) PAIRINGS

\begin{tabular}{c|c|c|c|c|c|c|c|c|c}
\hline Base-Station & \multicolumn{3}{|c|}{$B S_{1}$} & \multicolumn{3}{c|}{$B S_{2}$} & \multicolumn{3}{c}{$B S_{3}$} \\
\hline D2D Group/User & $U_{1}$ & $U_{2}$ & $U_{3}$ & $U_{4}$ & $U_{5}$ & $U_{6}$ & $U_{7}$ & $U_{8}$ & $U_{9}$ \\
\hline$D_{1}$ & $\checkmark$ & $\checkmark$ & $\checkmark$ & $x$ & $x$ & $x$ & $x$ & $x$ & $x$ \\
$D_{2}$ & $\checkmark$ & $\checkmark$ & $\checkmark$ & $x$ & $x$ & $x$ & $\checkmark$ & $\checkmark$ & $\checkmark$ \\
$D_{3}$ & $\checkmark$ & $\checkmark$ & $\checkmark$ & $\checkmark$ & $\checkmark$ & $\checkmark$ & $\checkmark$ & $\checkmark$ & $\checkmark$ \\
$D_{4}$ & $x$ & $x$ & $x$ & $\checkmark$ & $\checkmark$ & $\checkmark$ & $x$ & $x$ & $x$ \\
$D_{5}$ & $x$ & $x$ & $x$ & $\checkmark$ & $\checkmark$ & $\checkmark$ & $\checkmark$ & $\checkmark$ & $\checkmark$ \\
$D_{6}$ & $x$ & $x$ & $x$ & $x$ & $x$ & $x$ & $\checkmark$ & $\checkmark$ & $\checkmark$ \\
\hline
\end{tabular}

Furthermore, the transmit power per user channel is set to $P=1 \mathrm{~W}$, with noise variance $N_{0}=10^{-7} \mathrm{~W}$, and path-loss exponent is $\nu=3$. The target minimum SINR per user is set to ${ }^{8}$

\footnotetext{
${ }^{7}$ It should be noted that $D_{3}$ is the only D2D group that can potentially be paired to any of the $N=9$ users, since it falls within the overlapping area of all three base-stations.

${ }^{8}$ The target minimum SINR threshold per cellular user is set to be higher than that of the D2D receivers, since cellular users are assumed to have higher transmission priority, and thus must maintain higher QoS.
} 


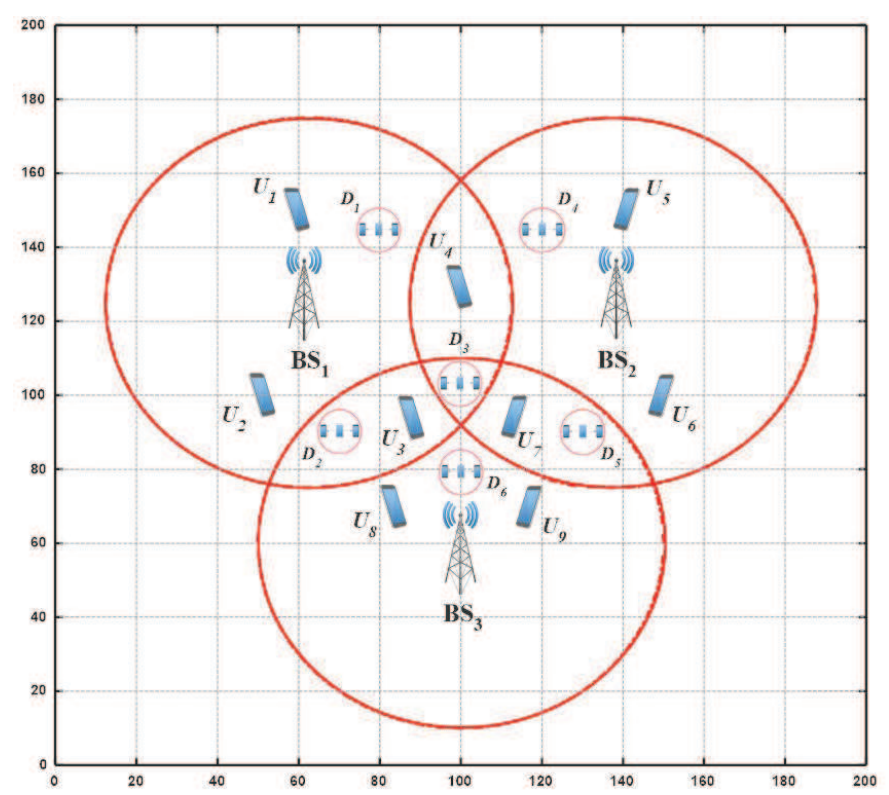

Fig. 2. Simulation Network Topology

$\bar{\gamma}_{T_{U}}=15 \mathrm{~dB}, \forall U_{n} \in \mathcal{U}$, while that for each D2D receiver is set to $\bar{\gamma}_{T_{D R}}=5 \mathrm{~dB}, \forall D_{m} \in \mathcal{D}$. The randomly generated channel coefficients are assumed to be quasi-static throughout the network operation, and are averaged over $5 \times 10^{3}$ independent network instances. Moreover, the following scenarios are considered:

Scenario 1: $\xi_{q}=1, \forall B S_{q} \in \mathcal{B}$.

Scenario 2: $\xi_{q}=3, \forall B S_{q} \in \mathcal{B}$.

In the above two scenarios, each base-station can be associated with at most one and three D2D groups, respectively. Thus, it would be expected that in the best-case scenario, at most three D2D groups may be paired with users under Scenario 1, but all six D2D groups may be paired with users under Scenario 2, provided the target minimum SINR constraints are satisfied. In addition, the two suggested scenarios aim at investigating the effect of increasing the number of D2D groups to be associated with each base-station on the resulting SINR of the users and D2D receivers, the percentage of $\mathrm{D} 2 \mathrm{D}$ association with each base-station, the percentage of identical stable matching solutions, the network sum-rate, and the number of iterations of the proposed stable matching algorithms. Last-but-not-least, the J-GA-CA-PA scheme ${ }^{9}$ is compared to the SP-DG-SM and SP-BS-SM schemes.

Remark 14: If a D2D group in any simulated network instance is not paired to a user channel (and thus not associated with a base-station), then the SINR at its receivers is set to zero.

\footnotetext{
${ }^{9}$ The J-GA-CA-PA scheme is solved with MIDACO [34], with tolerance set to 0.001 .
} 

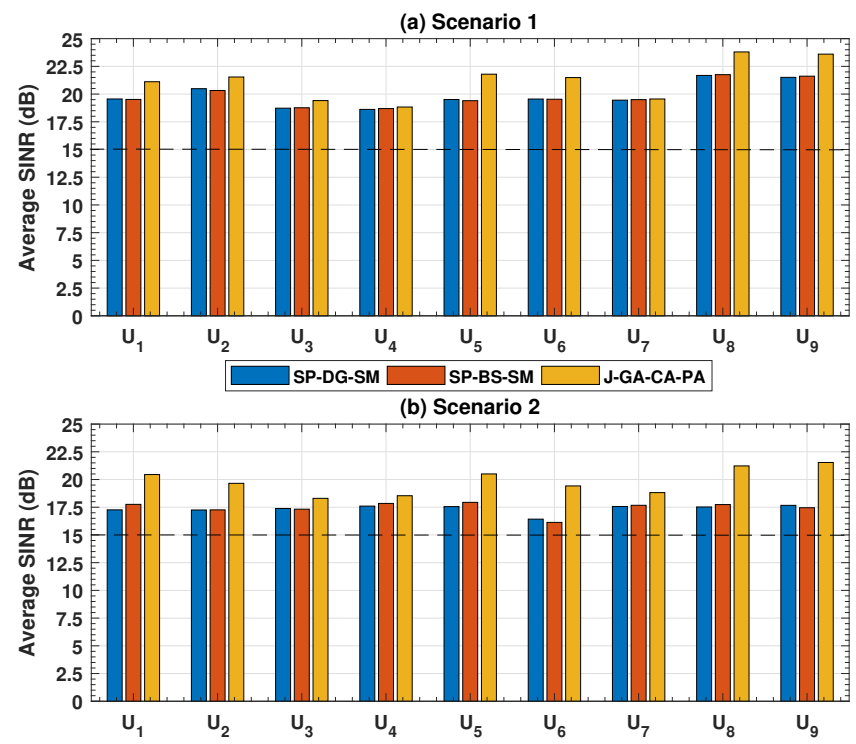

Fig. 3. Average SINR per Cellular User for: (a) Scenario 1, and (b) Scenario 2

Fig. 3 illustrates the average SINR per cellular user under Scenarios 1 and 2 for the SP-DGSM and SP-BS-SM schemes, and in comparison to the J-GA-CA-PA scheme. It is clear that all users satisfy the target minimum SINR of $\bar{\gamma}_{T_{U}}=15 \mathrm{~dB}$ under the aforementioned scenarios and schemes. More importantly, the SINR of each user under each matching algorithm is comparable to that under the J-GA-CA-PA. Remarkably, average SINR per user under the J-GA-CA-PA is never worse than any of the SP-DG-SM and SP-BS-SM, since it aims at maximizing the SINR of all cellular users and D2D receivers simultaneously without any bearing on stability (as per Remark 13). It is also noted that under Scenario 2, the average SINR per user is lower than under Scenario 1. This is due to the fact that under Scenario 2, more D2D groups are associated (paired to) with each base-station (user) on average. In turn, the available transmit power per user channel is shared more often between the D2D groups and users in Scenario 2 than in Scenario 1, and hence the reduction in the average SINR value per cellular user.

The average SINR of $\mathrm{D} 2 \mathrm{D}$ receiver $D R_{m_{1}}$ (i.e. the $\mathrm{D} 2 \mathrm{D}$ receiver with better channel conditions), $\forall D_{m} \in \mathcal{D}$, is illustrated in Fig. 4. It is evident that under Scenario 1 for both the SP-DG-SM and SP-BS-SM schemes and the J-GA-CA-PA scheme, $D R_{m_{1}}$ for D2D groups $D_{1}, D_{2}, D_{3}$, and $D_{5}$ satisfy the target minimum SINR of $\bar{\gamma}_{T_{D R}}=5 \mathrm{~dB}$, but this is not the case for D2D groups $D_{4}$ and $D_{6}$. This is due to the following reasons. First, D2D groups $D_{4}$ and $D_{6}$ may be paired to a cellular user less often than all the other groups (even if they satisfy their SINR threshold). This is due to their locations being relatively closer to $B S_{2}$ and $B S_{3}$, respectively, than the other D2D groups in their respective cells. Thus, they are more likely 

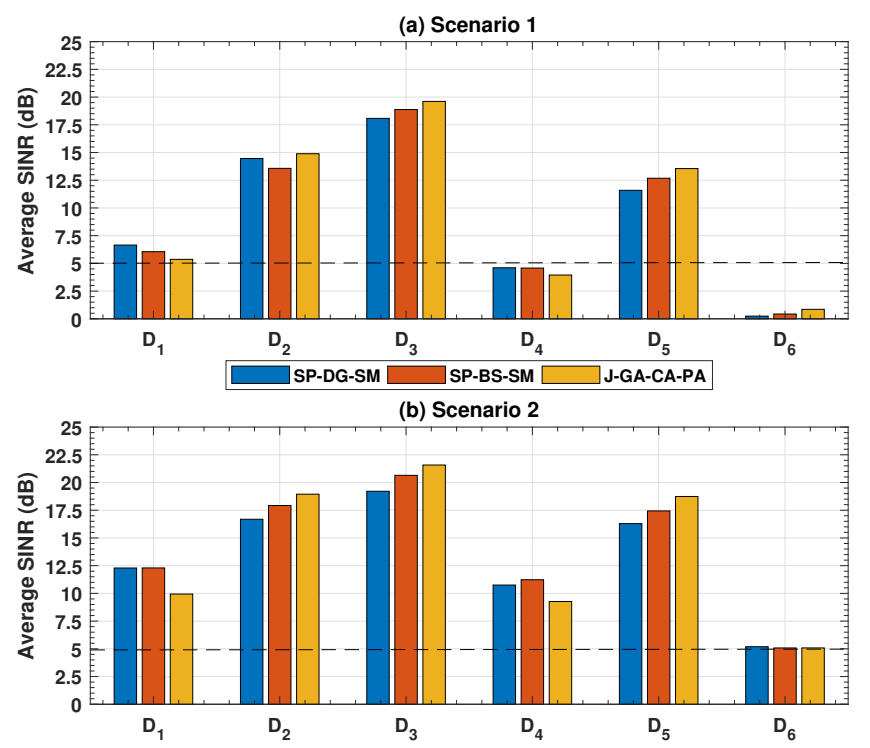

Fig. 4. Average SINR per D2D Receiver $D R_{m_{1}}$ for: (a) Scenario 1, and (b) Scenario 2

to cause higher interference to the users in their cells, which makes them less preferred than the other D2D groups. Also, every time D2D groups $D_{4}$ and $D_{6}$ are excluded from the stable matching solution of either algorithm, they achieve a SINR value of zero. This is also the case for the J-GA-CA-PA scheme, which excludes these two D2D groups if pairing them to a cellular user will prevent that cellular user from meeting its target minimum SINR. Second, since each base-station can be associated with a maximum of one D2D group under Scenario 1, then the other D2D groups that fall within the coverage area of $B S_{2}$ and $B S_{3}$ may be associated to them before D2D groups $D_{4}$ and $D_{6}$, and then no more D2D groups can be associated with $B S_{2}$ or $B S_{3}$. Contrarily, it is evident that $D R_{m_{1}}$ of D2D group $D_{3}$ achieves the highest SINR among all D2D groups. This is a direct result of its location being central to all three base-stations (i.e. it falls within the overlapping area of all base-stations). In turn, it has higher possibility of being paired to a user within any of the three cells. As for Scenario 2, it is clear that $D R_{m_{1}}$ for all D2D groups achieves the target minimum SINR. This implies that with the increase in the maximum number of D2D groups that can be associated with each base-station, D2D groups $D_{4}$ and $D_{6}$ are being associated more often with base-stations $B S_{2}$ and $B S_{3}$, respectively, which significantly improves their average SINR values. On the other hand, it is evident that the SINR values of $D R_{m_{1}}, \forall D_{m} \in \mathcal{D}$ are higher under Scenario 2 than Scenario 1. This is because under Scenario 2, more D2D groups can be associated with each base-station (and cellular user), which is interpreted as the D2D groups receiving zero SINR less often than in Scenario 1.

Fig. 5 demonstrates the average SINR of D2D receiver $D R_{m_{2}}$ (i.e. the D2D receiver with 

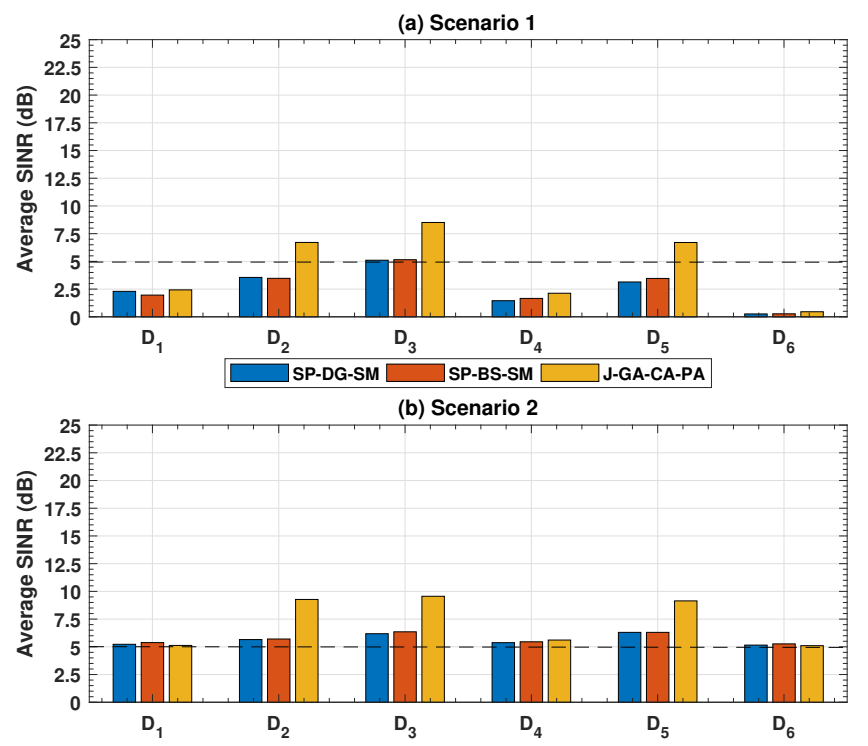

Fig. 5. Average SINR per D2D Receiver $D R_{m_{2}}$ for: (a) Scenario 1, and (b) Scenario 2

worse channel conditions), $\forall D_{m} \in \mathcal{D}$. Particularly, under Scenario 1, one can see that $D R_{m_{2}}$ of D2D group $D_{3}$ is the only receiver that meets the target minimum SINR of $5 \mathrm{~dB}$, for all SP-DGSM, SP-BS-SM, and J-GA-CA-PA schemes. However, under Scenario 2, D2D receiver $D R_{m_{2}}$ of all D2D groups achieve the target minimum SINR. As before, under Scenario 2, the average SINR is never worse than Scenario 1. Generally speaking, both $D R_{m_{1}}$ and $D R_{m_{2}}$ receivers of D2D groups $D_{2}, D_{3}$ and $D_{5}$ have relatively higher average SINR values than the other D2D groups, and this is because $D_{2}$ and $D_{5}$ fall into the overlapping region of two base-stations, while $D_{3}$ falls into the overlapping region of all three base-stations. In summary, a D2D group falling into the coverage area of two or more base-stations is more likely to be paired with a cellular user from any of the base-stations, which improves its average received SINR value.

In Fig. 6, the percentage of base-station association per D2D group under Scenario 1 is illustrated. It is clear that only $\mathrm{D} 2 \mathrm{D}$ groups $D_{1}, D_{2}$ and $D_{3}$ can be associated to base-station $B S_{1}$. Similarly, for base-station $B S_{2}$, only $\mathrm{D} 2 \mathrm{D}$ groups $D_{3}, D_{4}$ and $D_{5}$ can be associated to it. Moreover, $D_{2}, D_{3}, D_{5}$, and $D_{6}$ are the only D2D groups that can be associated with basestation $B S_{3}$ (i.e. all in alignment with Table II). It is also evident that the percentages of basestation association of the SP-DG-SM, SP-BS-SM and J-GA-CA-PA schemes are generally in agreement, with the discrepancy being the result of the different schemes do not necessarily yielding the same (D2D group, user) pairings, or D2D group and base-station associations. As for Scenario 2, similar observations can be made in Fig. 7. But more importantly, the average association of each D2D group under Scenario 2 is relatively higher than under Scenario 1. 


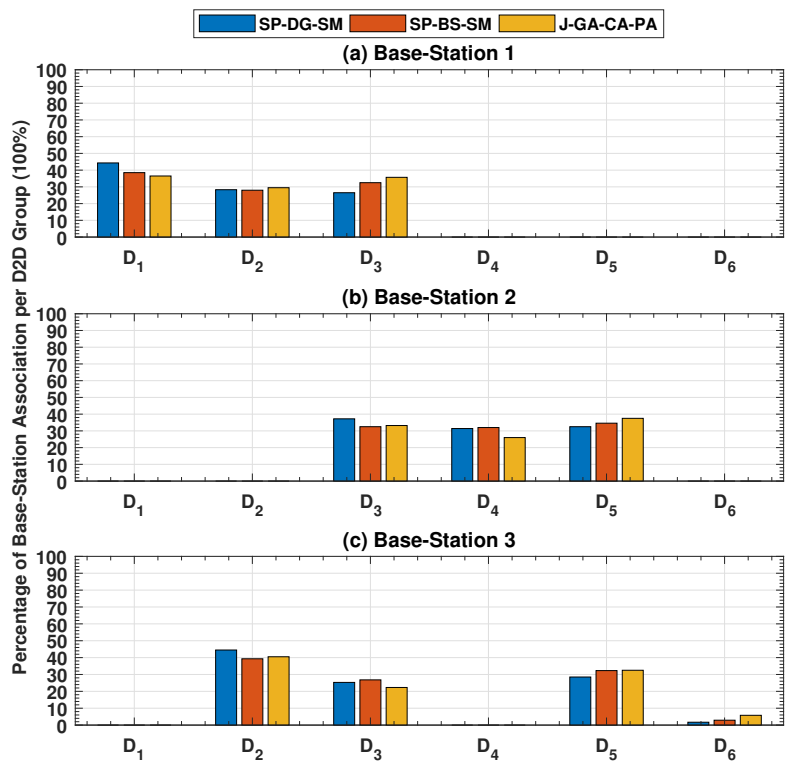

Fig. 6. Percentage of Base-Station Association per D2D Group Under Scenario 1 (\%100) for: (a) Base-Station 1, (b) Base-Station 2, and (c) Base-Station 3

This is because under Scenario 2, more D2D groups can be associated with each base-station (since $\xi_{q}=3, \forall B S_{q} \in \mathcal{B}$ ). Notably, the percentage of base-station association of D2D group $D_{6}$ has significantly improved in Scenario 2 in comparison to Scenario 1, which explains the improvement in the average SINR values of its receivers. Generally speaking, the matching algorithms themselves do not necessarily yield the same stable matching solutions, despite the use of the same solution procedure. This can be seen from Fig. 8, where under Scenario 1, about $\% 75$ of the time both algorithms yield the same stable matching solution; while under Scenario 2 both algorithms yield the same stable matching solution about \%42.5 of the time. This is attributed to the fact that the DG-SM algorithm yields D2D-group-optimal stable matching solutions, while the BS-SM algorithm yields base-station-optimal stable matching solutions (as per Lemma 4). Clearly, increasing the maximum number of D2D groups that can be associated with each base-station increases the possibility of obtaining different stable matching solutions.

Fig. 9 illustrates the percentage of D2D group unassignment under the different schemes and scenarios. Under Scenario 1, it can be seen from Fig. 9a that the SP-DG-SM and SP-BS-SM schemes yield comparable percentage of D2D group unassignment to that of the J-GA-CA-PA scheme. Moreover, it is evident that D2D group $D_{3}$ is the group with the least percentage of unassignment, which is followed by D2D groups $D_{2}$ and $D_{5}$. It is also clear that D2D group $D_{6}$ is unassigned the most, followed by $\mathrm{D} 2 \mathrm{D}$ groups $D_{4}$ and $D_{1}$, respectively. This explains the relatively lower average SINR for D2D receivers $D R_{m_{1}}$ and $D R_{m_{2}}$ for these groups, as shown 

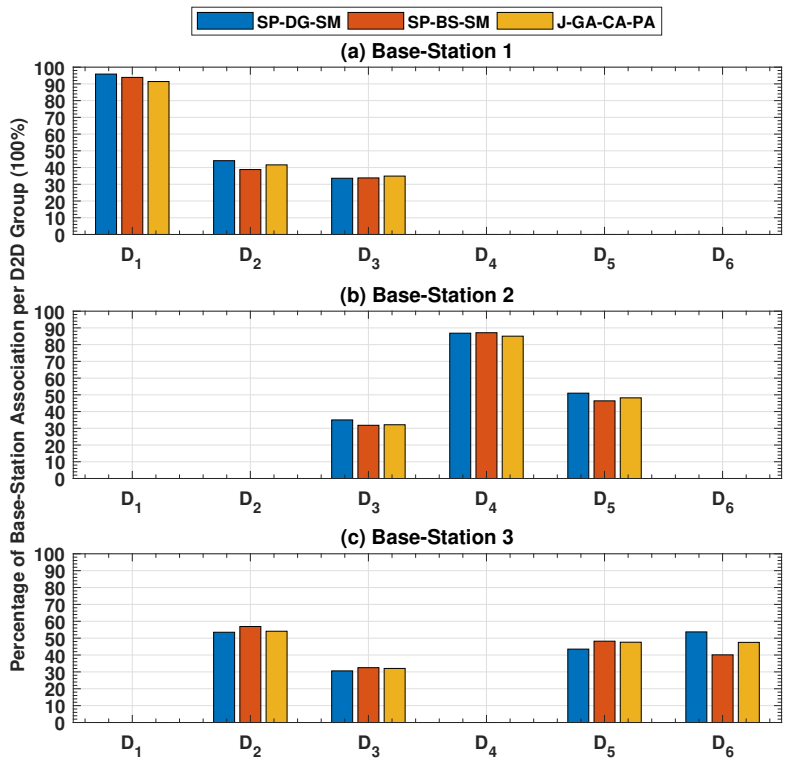

Fig. 7. Percentage of Base-Station Association per D2D Group Under Scenario 2 (\%100) for: (a) Base-Station 1, (b) Base-Station 2, and (c) Base-Station 3

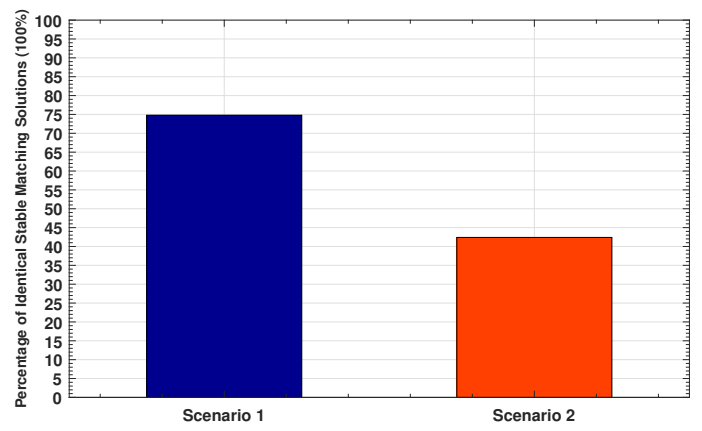

Fig. 8. Percentage of Identical Stable Matching Solutions (\%100)

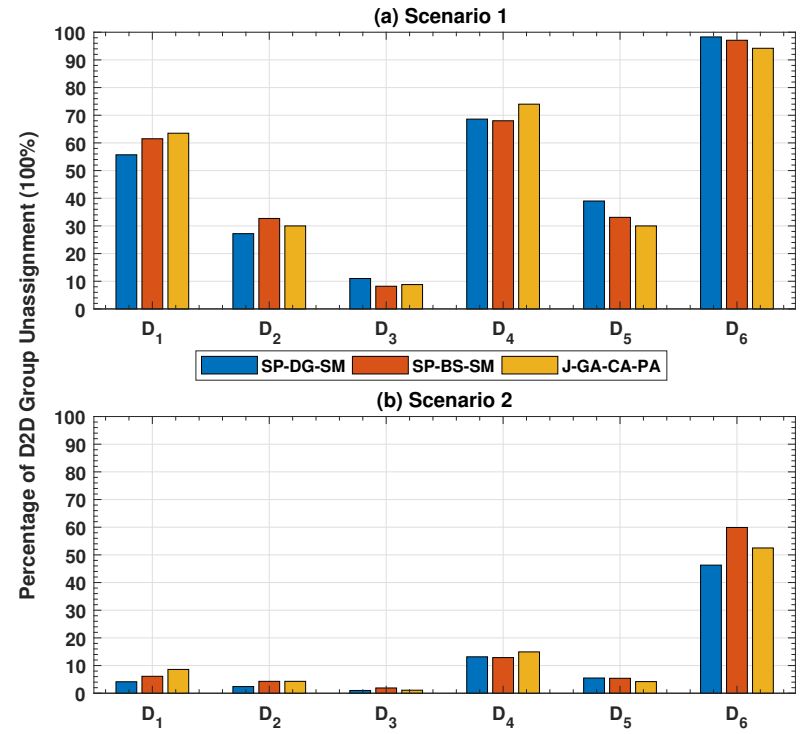

Fig. 9. Percentage of D2D Group Unassignment (\%100) for: (a) Scenario 1, and (b) Scenario 2 
in Figs. 4 and 5. Similar observations to Scenario 1 can be made under Scenario 2 (see Fig. 9b). Remarkably, the percentages of unassignment of D2D groups dropped significantly when compared to Scenario 1, and this is due to the fact that under Scenario 2, more D2D groups can be associated with each base-station.

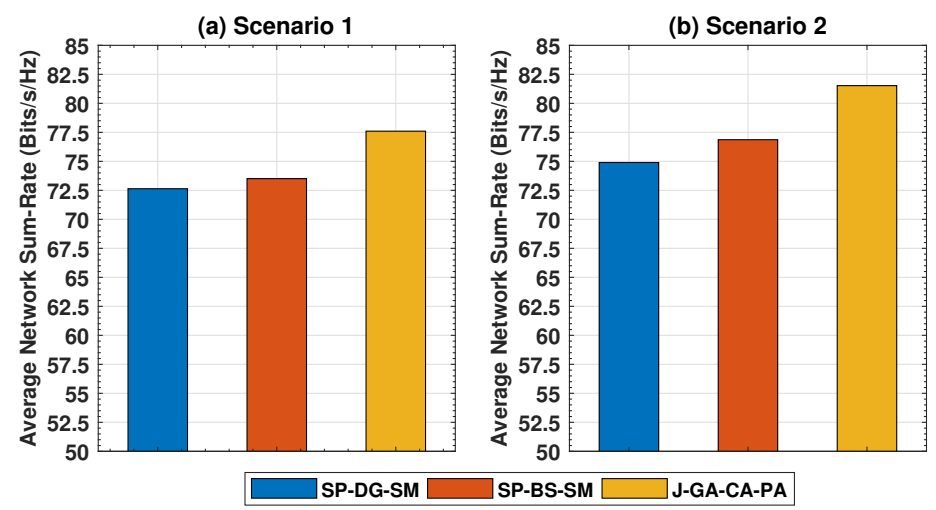

Fig. 10. Average Network Sum-Rate (Bits/s/Hz) for: (a) Scenario 1, and (b) Scenario 2

To shed light on the difference between the proposed SP-DG-SM and SP-BS-SM schemes in comparison to the J-GA-CA-PA scheme, Fig. 10 shows the average network sum-rate under Scenarios 1 and 2. Specifically, Fig. 10a illustrates the resulting average network sum-rate under Scenario 1, where it can be seen that the J-GA-CA-PA scheme is superior to the other two stable matching algorithms (at the expense of significantly higher computational complexity, as per Remark 4). Moreover, it is evident that the SP-BS-SM scheme yields slightly better average network sum-rate than its SP-DG-SM counterpart scheme. This also applies to Scenario 2, where a similar observation can be seen in Fig. 10b. It is also noticed that the network sum-rate of the different schemes is relatively higher in Scenario 2 than in Scenario 1. This is due to the fact that more D2D groups can be associated with base-stations, which improves the overall network sum-rate. It should be noted that the inferior sum-rate performance of the proposed matching algorithms in comparison to the J-GA-CA-PA is attributed to the following. Enforcing stable matching between the D2D groups and users is not necessarily optimal with respect to the network sum-rate (i.e. results in some sub-optimality in terms of the resulting SINR for the cellular users and the D2D groups). More specifically, a (D2D group, user) pair may form a blocking pair under any of the matching algorithms, and thus is eliminated from the resulting stable matching solutions; while that pair may be formed under the J-GA-CA-PA scheme, since it does not account for stability among the D2D groups and cellular users (as per Remark 13).

Last-but-not-least, in Fig. 11, the average number of iterations under the DG-SM and BS-SM algorithms for both scenarios is illustrated. It is evident that the DG-SM algorithm requires more 


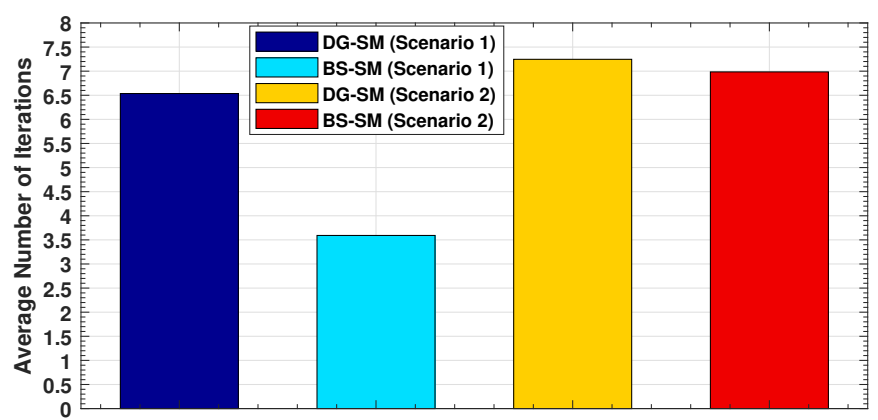

Fig. 11. Average Number of Iterations

iterations than the BS-SM algorithm, with the average number of iterations under Scenario 2 being slightly higher than under Scenario 1. Remarkably, both algorithms under both scenarios require on average less than 8 iterations, and thus can be executed efficiently. Moreover, for the simulated network topology, the SP-MO-SINR-MAX-PA requires a total of 9, 9, and 12 iterations at base-stations $B S_{1}, B S_{2}$, and $B S_{3}$, respectively, to determine the potential pairings for all possible (D2D group, user) pairs (as per Remark 10). Thus, the SP-MO-SINR-MAX-PA along with the proposed matching algorithms can be executed efficiently, while the J-GA-CAPA scheme is NP-complete (i.e. computationally-expensive), as per Remark 4. Based on the average network sum-rate results in Fig. 10, and the average number of iterations in Fig. 11, it can be concluded that for the simulated network topology, the SP-BS-SM scheme yields better network sum-rate, and requires less average number of iterations than the SP-DG-SM scheme. Lastly, the proposed matching algorithms with the SP-MO-SINR-MAX-PA significantly reduce the computational complexity_in comparison to the J-GA-CA-PA scheme-at the expense of some sub-optimality, which poses a reasonable tradeoff.

\section{CONCLUSIONS}

In this paper, the problem of joint D2D group association and channel assignment in uplink multi-cell NOMA networks has been studied. Specifically, a solution procedure for multiobjective SINR-maximizing power allocation has been devised to determine the preference of D2D groups over the potential cellular users, and also the preference of base-stations over the potential (D2D group, user) pairs, while accounting for interference and QoS requirements. After that, two polynomial-time complexity stable matching algorithms are proposed to associate D2D groups with base-stations and pair them with cellular users. The proposed matching algorithms and the devised solution procedure are shown to efficiently yield comparable SINR—per cellular user and D2D receiver-to the J-GA-CA-PA scheme, while maintaining QoS requirements. 


\section{ACKNOWLEDGEMENT}

\section{This work was partially supported by the Kuwait Foundation for the Advancement of Sciences} (KFAS) under project code PN17-15EE-02.

\section{REFERENCES}

[1] C. Xu, L. Song, and Z. Han, Resource Management for Device-to-Device Underlay Communication. Springer-Verlag (New York, NY, USA), 2013.

[2] A. Asadi, Q. Wang, and V. Mancuso, "A survey on device-to-device communication in cellular networks," IEEE Communications Surveys and Tutorials, vol. 16, no. 4, pp. 1801-1819, 2014.

[3] K. Doppler, M. Rinne, C. Wijting, C. B. Ribeiro, and K. Hugl, "Device-to-device communication as an underlay to LTE-advanced networks,” IEEE Communications Magazine, vol. 47, no. 12, pp. 42-49, Dec. 2009.

[4] X. Lin, J. G. Andrews, and A. Ghosh, "Spectrum sharing for device-to-device communication in cellular networks," IEEE Transactions on Communcations, vol. 13, no. 12, pp. 6727-6740, Dec. 2014.

[5] G. Fodor, E. Dahlman, G. Mildh, S. Parkvall, N. Reider, G. Miklos, and Z. Turanyi, "Design aspects of network assisted device-to-device communications," IEEE Communcations Magazine, vol. 50, no. 3, pp. 170-177, Mar. 2012.

[6] Z. Wei, J. Yuan, D. W. K. Ng, M. Elkashalan, and Z. Ding, "A survey of downlink non-orthogonal multiple accesss for 5G wireless communication networks," ZTE Communications, vol. 14, no. 4, pp. 17-25, Oct. 2016.

[7] Z. Ding, X. Lei, G. K. Karagiannidis, R. Schober, J. Yuan, and V. Bhargava, "A survey on non-orthogonal multiple access for 5G networks: Research challenges and future trends," IEEE Journal on Selected Areas in Communications, vol. 35, no. 10, pp. 2181-2195, Oct. 2017.

[8] W. Shin, M. Vaezi, B. Lee, D. J. Love, and H. V. Poor, "Non-orthogonal multiple access in multi-cell networks: Theory, performance and practical challenges," IEEE Communcations Magazine, vol. 55, no. 10, pp. 176-183, 2017.

[9] J. Zhao, Y. Liu, K. K. Choi, Y. Chen, M. Elkashlan, and J. Alonso-Zarate, "NOMA-based D2D communications: Towards 5G,” Proc. of IEEE Global Communications Conference (GLOBECOM), Dec. 2016.

[10] A. Anwar, B.-C. Seet, and X. J. Li, "Quality of service based NOMA group D2D communications," Future Internet, vol. 9, no. 73, pp. 1-14, 2017.

[11] Y. Pan, C. Pan, Z. Yang, and M. Chen, "Resource allocation for D2D communications underlaying a NOMA-based cellular network," IEEE Wireless Communications Letters, vol. 7, no. 1, pp. 130-133, Feb. 2018.

[12] S. M. A. Kazmi, N. H. Tran, T. M. Ho, A. Manzoor, D. Niyato, and C. S. Hong, "Coordinated device-to-device communication with non-orthogonal multiple access in future wireless cellular networks," IEEE Access, vol. 6, pp. 39 86039875 , Jun. 2018.

[13] J. Zhao, Y. Liu, K. K. Chai, Y. Chen, and M. Elkashlan, "Joint subchannel and power allocation for NOMA enhanced D2D communications," IEEE Transactions on Communications, vol. 65, no. 11, pp. 5081-5094, Nov. 2017.

[14] O. Semiari, W. Saad, S. Valentin, M. Bennis, and B. Maham, "Matching theory for priority-based cell association in the downlink of wireless small cell networks," Proc. of IEEE International Conference on Acoustics, Speech and Signal Processing (ICASSP), pp. 444-448, May 2014.

[15] O. Semiari, W. Saad, S. Valentin, M. Bennis, and H. V. Poor, "Context-aware small cell networks: How social metrics improve wireless resource allocation," IEEE Transactions on Wireless Communications, vol. 14, no. 11, pp. 5927-5940, Nov. 2015. 
[16] J. Zhao, Y. Liu, K. K. Chai, A. Nallanathan, Y. Chen, and Z. Han, "Spectrum allocation and power control for nonorthogonal multiple access in HetNets," IEEE Transactions on Wireless Communications, vol. 16, no. 9, pp. 5825-5837, Sept. 2017.

[17] B. Di, L. Song, and Y. Li, "Sub-channel assignment, power allocation and user scheduling for non-orthogonal multiple access networks," IEEE Transactions on Wireless Communications, vol. 15, no. 11, pp. 7686-7698, Nov. 2016.

[18] D. J. Abraham, R. W. Irving, and D. F. Manlove, "Two algorithms for the student-project allocation problem," Journal of Discrete Algorithms, vol. 5, no. 1, pp. 73-90, Mar. 2007.

[19] A. H. A. El-Atta and M. I. Moussa, "Student project allocation with preference lists over (student, project) pairs," Proc. of International Conference on Computer and Electrical Engineering (ICCEE), vol. 1, pp. 375-379, Dec. 2009.

[20] M. W. Baidas, M. S. Bahbahani, E. Alsusa, K. A. Hamdi, and Z. Ding, "D2D group association and channel assignment in uplink multi-cell NOMA networks," Proc. of IEEE Wireless Communications and Networking Conference (WCNC), Apr. 2019.

[21] F. Wang, W. Chen, H. Tang, and Q. Wu, "Joint optimization of user association, subchannel allocation, and power allocation in multi-cell multi-association OFDMA heterogenous networks," IEEE Transactions on Communications, vol. 65, no. 6, pp. 2672-2684, Jun. 2017.

[22] K. Mehmoud, M. T. Niaz, and H. S. Kim, "Dynamic fractional frequency resuse diversity design for intercell interference mitigation in nonorthogonal multiple access multicellular networks," Wireless Communications and Mobile Computing, pp. 1-18, 2018, DOI: 10.1155/2018/1231047.

[23] M. Ehrgott and X. Gandibleux, "An annotated bibliography of multiobjective combinatorial optimization," Report in Wissenschaftmathematik, vol. 62, 2000, fachbereich Mathematik, Universitat Kaiserslautern, Kaiserslautern, Germany.

[24] M. E.-A. Cherqui and M. Moulai, "An exact method for a discrete multiobjective linear fractional optimization,” Journal of Applied Mathematics and Decision Sciences, vol. 12, 2008, DOI: 10.1155/2008/760191.

[25] Y. Collette and P. Siarry, Multiobjective Optimization: Principles and Case Studies. Springer, 2013.

[26] T. Rapcsak, “On pseudolinear functions,” European Journal of Operations Research, vol. 50, pp. 353-360, 1991.

[27] A. Cambini and L. Martein, Generalized Convexity and Optimization: Theory and Applications. Springer Science \& Business Media, 2008.

[28] S. Schaible, Handbook of Global Optimization: Fractional Programming. Kluwer Academic Publishers, DordrechtBoston-London, 1995.

[29] S. Schaible and J. Shi, "Recent developments in fractional programming: Single ratio and max-min case," Proc. of International Conference on Nonlinear Analysis and Convex Analysis, pp. 493-506, 2003.

[30] N. Guzel, "A proposal to the solution of multiobjective linear fractional programming problem," Abstract and Applied Analysis, vol. 11, 2013, DOI: 10.1155/2013/435030.

[31] S. Boyd and L. Vandenberghe, Convex Optimization. Cambridge University Press, 2003.

[32] Q.-T. Vien, T. A. Le, B. Barn, and C. V. Phan, "Optimising energy efficiency of non-orthogonal multiple access for wireless backhaul in heterogenous cloud radio access network," IET Communications, vol. 10, no. 18, pp. 2516-2524, Dec. 2016.

[33] H. Q. Tran, P. Q. Truong, C. V. Phan, and Q.-T. Vien, "On the energy efficiency of NOMA for wireless backhaul in multitier heterogenous CRAN," Proc. of International Conference on Recent Advances in Signal Processing, Telecommunications and Computing (SigTelCom), pp. 229-234, Feb. 2017.

[34] M. Schlueter, "MIDACO software performance on interplanetary trajectory benchmarks," Advances in Space Research, vol. 54, no. 4, pp. 744-754, 2014. 